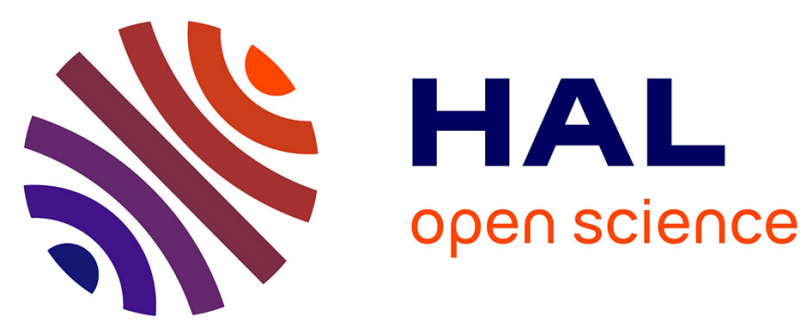

\title{
It makes sense, so I see it better! Contextual information about the visual environment increases its perceived sharpness.
}

Pauline Rossel, Carole Peyrin, Alexia Roux-Sibilon, Louise Kauffmann

\section{To cite this version:}

Pauline Rossel, Carole Peyrin, Alexia Roux-Sibilon, Louise Kauffmann. It makes sense, so I see it better! Contextual information about the visual environment increases its perceived sharpness.. Journal of Experimental Psychology: Human Perception and Performance, inPress, 10.1037/xhp0000993 . hal-03564822

\section{HAL Id: hal-03564822 \\ https://hal.science/hal-03564822}

Submitted on 10 Feb 2022

HAL is a multi-disciplinary open access archive for the deposit and dissemination of scientific research documents, whether they are published or not. The documents may come from teaching and research institutions in France or abroad, or from public or private research centers.
L'archive ouverte pluridisciplinaire HAL, est destinée au dépôt et à la diffusion de documents scientifiques de niveau recherche, publiés ou non, émanant des établissements d'enseignement et de recherche français ou étrangers, des laboratoires publics ou privés. 
PRIOR SCENE KNOWLEDGE INCREASES ITS PERCEIVED SHARPNESS

This is the unformatted version of the paper:

Rossel, P., Peyrin, C., Roux-Sibilon, A., \& Kauffmann, L. (2022). It makes sense, so I see it better! Contextual information about the visual environment increases its perceived sharpness. Journal of Experimental Psychology: Human Perception and Performance. Advance online publication. https://doi.org/10.1037/xhp0000993

(C) 2022, American Psychological Association. This paper is not the copy of record and may not exactly replicate the final, authoritative version of the article. Please do not copy or cite without authors' permission. The final article will be available, upon publication, via its DOI: 10.1037/xhp0000993

It Makes Sense, so I See it Better! Contextual Information About the Visual Environment Increases its Perceived Sharpness

Pauline Rossel $^{1}$, Carole Peyrin ${ }^{1}$, Alexia-Roux-Sibilon ${ }^{2}$ and Louise Kauffmann ${ }^{1}$

${ }^{1}$ CNRS, LPNC, Univ. Grenoble Alpes, Univ. Savoie Mont Blanc, Grenoble, France

${ }^{2}$ Psychological Sciences Research Institute (IPSY), UC Louvain, Louvain-la-Neuve, Belgium

Author Note

We have no known conflict of interest to disclose.

This study was not preregistered but stimuli, executable files of the experiment, data and analysis scripts are available in the Open Science Framework repository at https://osf.io/d65zs/. 
PRIOR SCENE KNOWLEDGE INCREASES ITS PERCEIVED SHARPNESS

Correspondence concerning this article should be addressed to Louise Kauffmann, Univ. Grenoble Alpes, Univ. Savoie Mont Blanc, Grenoble, France. E-mail: louise.kauffmann@univ-grenoble-alpes.fr

\begin{abstract}
Predictive coding theories of visual perception postulate that expectations based on prior knowledge modulate the processing of information by sharpening the representation of expected features of a stimulus in visual cortex but few studies directly investigated whether expectations qualitatively affect perception. Our study investigated the influence of expectations based on prior experience and contextual information on the perceived sharpness of objects and scenes. In Experiments 1 and 2, we used a perceptual matching task. Participants saw two blurred images depicting the same object or scene and had to adjust the blur level of the right image to match the blur level of the left one. We manipulated the availability of relevant information to form expectations about the image's content: one of the two images contained predictable information while the other one unpredictable. At an equal level of blur, predictable objects and scenes were perceived as sharper than unpredictable ones. Experiment 3 involving explicit sharpness judgments confirmed these results. Our findings support the sharpening account of predictive coding theories by showing that expectations increase the perceived sharpness of the visual signal. Expectations about the visual environment help us understand it more easily, but also makes us perceive it better.
\end{abstract}

Keywords: predictive coding, expectations, visual perception, perceptual matching, sharpening 


\section{PRIOR SCENE KNOWLEDGE INCREASES ITS PERCEIVED SHARPNESS}

Many studies have shown that what we know or expect based on past experience and contextual information can influence how easily we can see and interpret the visual world: for example, a cow is recognized faster when presented in a field than a kitchen context or in isolation. Here we show that expectations also affect how we see. In three experiments, we found that blurred scenes and objects that could be related to prior knowledge (upright scenes and objects in predictable context) were perceived as sharper than the same blurred scenes and objects that could not (inverted scenes and objects in unpredictable contexts). Prior knowledge and expectations about the visual environment therefore not only help us understand it more easily, but also makes us perceive it better. 
PRIOR SCENE KNOWLEDGE INCREASES ITS PERCEIVED SHARPNESS

\section{It Makes Sense, so I See it Better! Contextual Information About the Visual Environment Increases its Perceived Sharpness}

\section{Introduction}

Making sense of the visual world first necessitates solving a perceptual problem. The light casts shadows on objects, which often overlap and can have an infinity of appearances depending on viewpoints or lightning conditions. Yet, we are able to recognize objects and scenes in a few hundred milliseconds despite their complexity and their variability (Kirchner \& Thorpe, 2006; Rousselet et al., 2005; Thorpe et al., 1995). In order to deal with this problem and achieve recognition, we strongly rely on expectations based on prior experience and regularities learnt from the environment. For instance, we always see objects in context in dayto-day experience. We can therefore create associations between objects and their contexts leading to expectations or predictions about which objects could appear in a particular context or in which contexts a specific object is likely to be found (Bar, 2004, 2007; Oliva \& Torralba, 2007). By restricting the possible interpretations of the stimulus we are perceiving, these expectations would facilitate the processing of visual information that fits them (Bar, 2004, 2007; Roux-Sibilon et al., 2019).

Many studies have shown that a predictable stimulus in a given context (e.g., a priest in a church) is recognized more accurately or more quickly than a non-predictable one (e.g., a priest on a football field; (Brandman \& Peelen, 2017; Davenport, 2007; Davenport \& Potter, 2004; Greene et al., 2015; Joubert et al., 2008; Lai et al., 2020; Palmer, 1975; Roux-Sibilon et al., 2019). In that sense, expectations about the visual environment influence how easily we perceive it. Expectations would be particularly useful to enable the recognition of noisy visual stimuli, for which the mere analysis of the input's physical attributes may be inefficient. For 


\section{PRIOR SCENE KNOWLEDGE INCREASES ITS PERCEIVED SHARPNESS}

example, two blurry light points on a foggy road can only be interpreted as a car coming from the opposite direction if one relies on prior knowledge and learned associations about the objects that can be found in such a context. Expectations based on contextual information can even influence what we perceive when the visual signal is ambiguous. For example, a dark elliptical shape appearing in a blurry street context can be either interpreted as a car or as a pedestrian depending on its orientation (Oliva \& Torralba, 2007; Torralba, 2003; see also Bar, 2004; Roux-Sibilon et al., 2019). Current models of visual perception therefore consider visual processing as a proactive process, where conscious perception is not only determined by the characteristics of sensory inputs, but also strongly depends on expectations about them (Bar, 2007; Clark, 2013; de Lange et al., 2018; Friston, 2005; Summerfield \& Egner, 2009; Teufel $\&$ Fletcher, 2020). This assumption more broadly falls within the cognitive penetrability of perception theoretical framework, according to which perception influences cognition, but is also constrained by it (Lupyan, 2015; O'Callaghan et al., 2017).

Yet, evidence that expectations qualitatively and subjectively affect perception is still scarce. Indeed, past studies investigating the effects of expectations mainly focused on implicit measures such as reaction times during recognition tasks which may not be sufficient to address subjective perceptual effects. For example, the fact that an expected stimulus is recognized faster than an unexpected one suggests an influence of expectations on processing speed but it does not necessarily follow that this is also associated with a qualitative change in perception. Similarly, higher recognition rate of an expected compared to an unexpected stimulus could be achieved by guessing the identity of the expected stimulus and does not necessarily reflect its enhanced perception. Behavioral tasks involving subjective perceptual reports are therefore needed to address whether expectations influence subjective perception.

Another important question is how expectations do so. In this respect, the predictive coding theoretical framework (Rao \& Ballard, 1999; see also Friston, 2005; Spratling, 2017; 


\section{PRIOR SCENE KNOWLEDGE INCREASES ITS PERCEIVED SHARPNESS}

for reviews see de Lange et al., 2018; Kok \& de Lange, 2015) provides useful insights. This model assumes that perception results from a continuous exchange of Prediction and Prediction Error signals between adjacent levels of the cortical hierarchy. Prediction signals are transmitted via descending connections and represent the current hypotheses that best explain the input one area receives. Prediction error signals carry the mismatch between these predictions and the actual input information. They are sent via ascending connections to the next upper cortical area allowing to adjust the predictions so that they better fit the input. Moreover, the relative weight of Prediction and Prediction error signals would vary according to the reliability of the visual signal: Prediction signals would weight more when the stimulus is noisy or ambiguous while Prediction error signals would weight more when the stimulus is unambiguous (e.g., Feldman \& Friston, 2010; Press et al., 2020). Different theories have been proposed regarding the neural implementation of predictive coding. The dampening account (also referred to as the Cancellation theory) posits that prediction signals are used to filter out or explain-away the predicted features of a stimulus, by inhibiting neurons tuned to these features, resulting in a relatively higher activity of neurons tuned to the unexpected features (e.g., Blakemore et al., 1998; Murray et al., 2004). Alternatively, the sharpening account (also referred to as the Bayesian theory) assumes that prediction signals increase the sensitivity of neurons tuned to the expected features while suppressing the response of neurons tuned to unexpected ones, resulting in a sharper cortical representation of expected stimuli (e.g., Kaiser et al., 2019; Lee \& Mumford, 2003). To date, results from neuroimaging experiments have provided contradictory findings arguing in favour of both accounts (see de Lange et al., 2018 for a review). For example, Kok et al. (2012) presented participants with gratings whose orientation could be predicted or not based on auditory cues in an fMRI experiment. By examining the pattern of activity (rather than the mean activation) in the primary visual cortex using multivoxel pattern analyses, they found that the orientation of the grating could be 
PRIOR SCENE KNOWLEDGE INCREASES ITS PERCEIVED SHARPNESS

decoded with higher accuracy from V1's activity pattern when it was expected than unexpected. These results therefore supported the view that expectations sharpen representations in the visual cortex. Using a similar decoding method in the macaque inferotemporal cortex, Kumar et al. (2017) however found the opposite. Expected stimuli were decoded less accurately than unexpected ones, arguing in favour of the dampening account. It has also been proposed that both mechanisms can coexist in separate neural populations or take place at different times over the course of perception (de Lange et al., 2018; Friston, 2005; Press et al., 2020).

While perception may not truly reflect the neural computations occurring in sensory cortices, hypotheses can be derived from these two mechanisms about the perceptual consequences of expectations. The dampening account should result in increasing the sensitivity to unexpected features which should be better detected than expected ones. On the contrary, the sharpening account should result in a higher sensitivity to expected than unexpected features. Recent behavioural studies tend to support the latter hypothesis (Alilović et al., 2021; Cheadle et al., 2015; Han \& VanRullen, 2016; Lupyan, 2017; Perez et al., 2020; Stein \& Peelen, 2015; Teufel et al., 2018; Wyart et al., 2012) by showing that expectations, or prior knowledge about a visual stimulus (e.g., familiar objects or meaningful shapes), increase the sensitivity to features matching these expectations by enhancing their detection. For example, Teufel et al. (2018) asked participants to detect the contrast or orientation of Gabor patches embedded in meaningless two-tone ("Mooney") images. Participants were then shown the original - meaningful - images used to build the two-tone stimuli and completed the task again. The authors observed that semantic knowledge about the images improved orientation and contrast detection during the second session, only when the border of Gabor patches matched the location of contours in the original images which were no longer present in the two-tone images. These results therefore indicated that - even if irrelevant to the task - object 


\section{PRIOR SCENE KNOWLEDGE INCREASES ITS PERCEIVED SHARPNESS}

knowledge enhances the detection of consistent low-level features. However, only a few studies used explicit perceptual reports to directly address whether expected stimuli actually look sharper (Han \& VanRullen, 2016; Lupyan, 2017; Perez et al., 2020). Of particular interest, a study by Lupyan (2017) showed that prior semantic knowledge has a direct impact on perceived sharpness of the visual signal. In this experiment, participants completed a perceptual matching task in which they were presented with two blurred letter strings, one being the Target and the other one the Sample. Their task was to adjust the blur level of the Sample in order to match the blur level of the Target. To address the role of prior knowledge on the perceived sharpness of stimuli, the meaningfulness of the Target/Sample letter strings was manipulated so that one of the strings was a meaningful word (prior semantic knowledge, e.g., "much") and the other one a meaningless pseudoword obtained via shuffling the order of the meaningful word's letters (no prior semantic knowledge, e.g., "mchu"). The Target could be a word and the Sample a pseudoword or the Target could be a pseudoword and the Sample a word. Results showed that participants added more blur than necessary to match a Sample word to a Target pseudoword and not enough blur to match a Sample pseudoword to a Target word. This indicated that at an objectively equal level of blur, words were subjectively perceived to be sharper than pseudowords. These results suggested that expectations based on prior semantic knowledge influenced the processing of blurred meaningful words by making them look sharper, and therefore that expectations also affect how we perceive.

Such a mechanism may be particularly useful for the processing of visual stimuli such as words requiring a detailed processing, and therefore, a sharp percept. However, does it also apply to the processing of visual stimuli such as objects in context or even whole scenes which need less details to be identified (Greene \& Oliva, 2009; Kauffmann et al., 2014; Schyns \& Oliva, 1994)? This question is crucial to make it a more general principle of visual perception. 


\section{PRIOR SCENE KNOWLEDGE INCREASES ITS PERCEIVED SHARPNESS}

The aim of the present study was therefore to determine whether prior knowledge about our visual environment can affect the appearance of objects and scenes.

To this end, we used a perceptual matching task inspired by Lupyan's paradigm (2017). This task appears particularly relevant to address the consequences of expectations on subjective perception because it allows to directly compare participants' own perceptual judgments to the known "true state" of the signal. Any resulting difference is thus more likely to reflect qualitative perceptual effects, in contrast to measures such as reaction times or recognition accuracy. In Experiments 1 and 2, two blurred images depicting a scene, a Target and a Sample, were displayed side by side on the screen. Participants had to adjust the blur level of the Sample in order to match the blur level of the Target. As in Lupyan (2017), we manipulated the availability of prior experience or relevant information to form expectations about the image content. In Experiment 1, we addressed the effect of expectations on the perceived sharpness of an object by manipulating its scene context. The object was either surrounded by its intact scene context (predictable object) or by a phase-scramble of its scene context (unpredictable object). The Sample and the Target always contained the same object but had a different context. In Experiment 2, we addressed the effect of expectations on the perceived sharpness of a whole scene by manipulating the predictability of scene layout. The scene was either presented upright (predictable scene layout) or upside-down (unpredictable scene layout). The Sample and the Target were images of the same scene but with a different orientation. Based on Lupyan's results (2017), we expected stimuli to be perceived as sharper in predictable than unpredictable conditions. Therefore, at an objectively equal blur level, predictable stimuli should be subjectively perceived to be sharper than unpredictable ones in both experiments. As a consequence, participants should match the blur level of the Target and the Sample stimuli by attributing to a predictable Sample matched to an unpredictable Target a higher blur level than to an unpredictable Sample matched to a predictable Target. 
PRIOR SCENE KNOWLEDGE INCREASES ITS PERCEIVED SHARPNESS

\section{Experiment 1}

\section{Method}

\section{Participants and Design}

Thirty-two undergraduate students of Psychology from University Grenoble Alpes (28 women, $\mathrm{M}_{\mathrm{age}}=22.38, \mathrm{SD}_{\mathrm{age}}=4.84$ ) with normal or corrected to normal vision participated in the experiment. The sample size was set based on an estimated effect size of $d_{z}=0.62$ from Experiment 1B of Lupyan (2017) to achieve a power (i.e., corresponding to 1 - $\beta$, with $\beta$ being the risk of Type- 2 error) of 0.8 with an alpha level of 0.05 . To estimate this effect size, we calculated the Cohen's $\mathrm{d}_{\mathrm{z}}$ from the $t$ statistic of the interaction between the predictability of the Target and the type of trial. Then we calculated the confidence interval from this Cohen's $d_{z}$ and we selected the lower bound. The study was approved by the ethics committee of the University Grenoble Alpes (CER-Grenoble Alpes, COMUE University Grenoble Alpes, CERGA-Avis-2020-4) and was conducted in agreement with the Declaration of Helsinki for experiments involving human subjects. All participants involved in the study gave their informed consent before taking part in the experiment. They received course credits for their participation.

\section{Stimuli}

The stimuli were constructed from 20 coloured photographs of real-world scenes (10 indoor scenes and 10 outdoor scenes) downloaded from the Pixabay website (https://pixabay.com/fr/), a photo sharing site under Creative Commons Zero licence. The stimuli were created using MATLAB R2019b (The Mathworks, Natick, MA). Original images were cropped to a square format and resized to $350 \times 350$ pixels. They were converted to 256 level grey-scale images by averaging the values from the three colour channels for each pixel and the mean luminance of all images was equalized to 0.5 (for pixel intensity values between 0 and 1). 
PRIOR SCENE KNOWLEDGE INCREASES ITS PERCEIVED SHARPNESS

Each scene contained an object semantically related to the scene category (e.g., a car for an outdoor scene, a lamp for an indoor scene) which was delineated by an ellipse encompassing $176 \times 166$ pixels of vertical and horizontal diameters. The object could be at different locations according to the scene image. For each scene, we created two versions: one in which the scene context around the object was left intact (predictable object based on contextual information, Intact condition) and one in which it was phase-scrambled and therefore did not contain semantic information (unpredictable object based on contextual information, Scrambled condition). The phase-scrambled version of the scene was built by combining the amplitude spectrum of the scene with the phase spectrum of a white noise in Fourier space and the resulting image was obtained via inverse Fourier Transform.

The ellipse containing the object was then blurred using 2-D Gaussian filters (imgaussfilt Function in Matlab) of linearly increasing size (i.e., increasing standard deviation - SD) in order to obtain 31 versions of the object (Figure 1) ranging from a sharp object (close to intact; blur level $1, \mathrm{SD}=1$ ) to a very blurred and hardly recognizable one (blur level $31, \mathrm{SD}$ $=10$ ). Blurring of stimuli had no effect on the mean luminance of stimuli which was set to 0.5 (for pixels intensities between 0 and 1) prior to applying the gaussian filters. It only resulted in slightly reducing the root mean squared (RMS) contrast of stimuli (from 0.189 at blur level 1 to 0.185 at blur level 31 ). For each blur level, the ellipse containing the object was then added on the intact and scrambled scene backgrounds and was superimposed on a larger grey elliptical frame of average luminance ( 0.5 for pixel intensity values between 0 and 1 ) and 30 pixels wide. This grey frame allowed us to isolate the object from the background and avoid local contrast differences between the object and the intact or scrambled backgrounds.

The integration of the object and grey frame on intact and scrambled backgrounds was done as follows. To integrate the grey elliptical frame to the image background, we first created for each stimulus a binary mask of the elliptical frame. This mask consisted of an image of 350 
PRIOR SCENE KNOWLEDGE INCREASES ITS PERCEIVED SHARPNESS

$\times 350$ pixels (same resolution as the scenes) containing an ellipse of $236 \times 226$ pixels of vertical and horizontal diameters centred on the object location. Pixel values within the ellipse were set to 1 while pixel values outside of the ellipse were set to 0 . This binary mask was then spatially filtered by a gaussian with a standard deviation of 2 to smooth the border of the elliptical frame. The resulting image was multiplied with average luminance values of 0.5 to fill the ellipse with a uniform grey. We then computed the inverse of the smoothed binary mask of the ellipse to obtain an image with pixel values of 0 within the ellipse and 1 in the background. This inverse mask was multiplied with the intact or scrambled scene resulting in an image containing the scene background and pixel values set to 0 within the ellipse. Finally, this image was added to the image containing the grey elliptical frame. A similar procedure was used to blend the smaller ellipse containing the object with the background and the grey elliptical frame. We first created a binary mask of an ellipse of $176 \times 166$ pixels of vertical and horizontal diameters centred at the object location which was then spatially smoothed by a gaussian with a standard deviation of 2 . The resulting image was multiplied with the original scene to obtain an image containing only the object (pixel values outside of the ellipse were set to 0 ). We then created the inverse of the smoothed binary mask and the resulting image was multiplied with the scene containing the grey elliptical frame. The resulting image was added to the image containing the elliptical object to obtain the final stimulus.

\section{Figure 1}

Examples of an Object at Different Blur Levels Between Blur Levels 1 and 31 in the Intact and Scrambled Conditions 


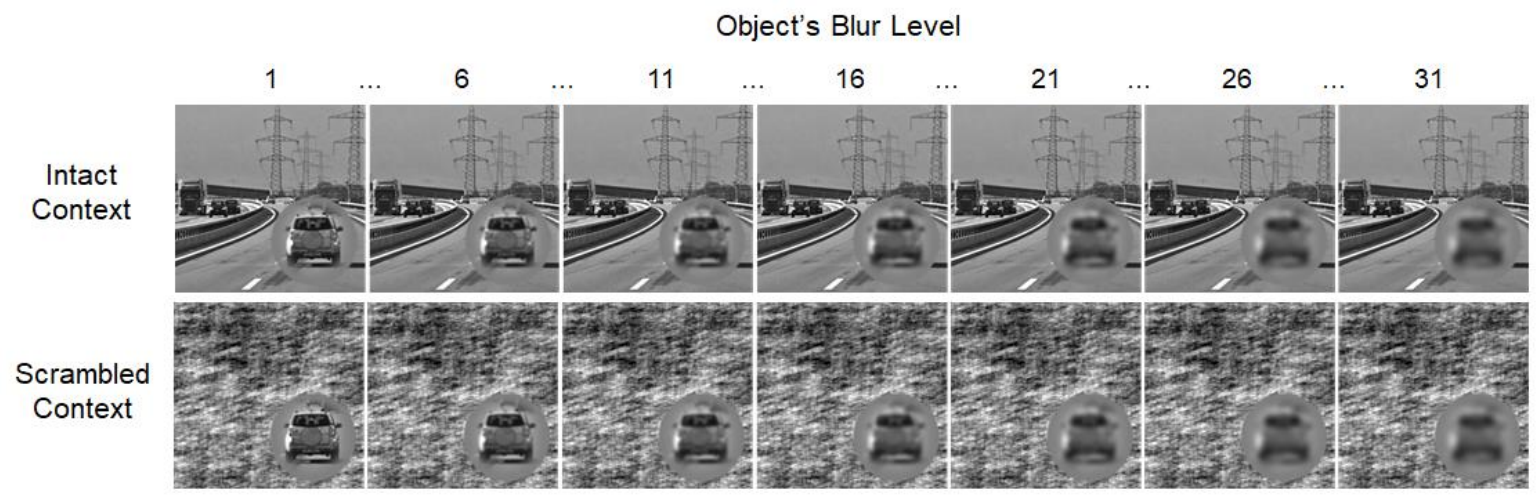

\section{Procedure}

The experiment was performed in the context of the Covid-19 lockdown periods in France and was therefore conducted online. It was constructed using E-Prime 3.0 software (EPrime Psychology Software Tools Inc., Pittsburgh, USA) and stimuli were displayed using an E-Prime Go 1.0 executable file on the participant's personal computer. Before starting the experiment, the experimenter met each participant via videoconference in order to explain the task and ensured that basic computer criteria were met to perform the experiment (e.g., an operating system supporting E-prime Go, screen resolution of $1920 \times 1080$ pixels ensuring the correct display of stimuli).

On each trial, two images containing the same object were displayed on the left and right of the participant's computer screen against a grey background (average luminance of 0.5). The object in the left image was the Target (T) and the object in the right image was the Sample (S). The Target object was presented with a randomly selected blur level between level 6 and 25 which remained constant throughout the trial. The Sample object always had an initial blur level of 16 (median blur level between levels 1 and 31) at the beginning of the trial. Participants were instructed to adjust the blur level of the Sample object to match the blur of the Target object (Figure 2) by increasing or decreasing the blur of the Sample object in 1-level steps using the "P" and "O" keys on their keyboard, respectively. The Sample could be adjusted up to a maximum blur corresponding to level 31 and a minimum blur corresponding to level 1 . 
PRIOR SCENE KNOWLEDGE INCREASES ITS PERCEIVED SHARPNESS

Participants had to press the "Y" key to validate their response once they judged the Sample and Target objects as equally blurred. Participants were encouraged to rely on their subjective judgment to match the blur level of objects.

For each trial, the backgrounds of each object could be either intact (predictable object) or phase-scrambled (unpredictable object). Furthermore, the Target (T) and Sample (S) objects either had different (different Target/Sample pair-types) or the same background (identical Target/Sample pair-types) resulting in four conditions (see Figure 2a). The different Target/Sample pair-types allowed us to assess the effect of contextual information on the perceived sharpness of objects. The identical Target/Sample pair-types were used as control conditions to assess any general tendency in over- or underestimating the blur of the Sample relative to the Target object irrespective of its predictability, or to detect a more systematic bias in underestimating the blur of objects embedded in intact contexts irrespective of what they are being matched to. The predictable and unpredictable conditions manipulated the presence and absence of semantic information to generate expectations about the object, but the context (either intact or scrambled) was never relevant to carry out the task which consisted in the blur level adjustment of the object. This clarification is important to dissociate the effects of expectations (i.e., what is likely to be present in the signal) from effects of attention (i.e., what is relevant for the task at hand) as attention has been shown to enhance perception (Rungratsameetaweemana \& Serences, 2019; Summerfield \& de Lange, 2014).

An experimental session contained 160 trials appearing in a random order (20 objects presented twice in each of the four conditions) with a break in approximately the first and second third of the experiment. For all conditions, each of the 20 Target objects appeared with a blur level varying from 6 to 25 (i.e., a total of 20 blur levels) so that each blur level was presented once per condition for a randomly selected object. The experiment lasted about 3040 minutes, depending on the speed at which each participant made their judgment (mean \pm 
PRIOR SCENE KNOWLEDGE INCREASES ITS PERCEIVED SHARPNESS

SD duration of a trial: $1858 \pm 2133 \mathrm{~ms}$ ). For each trial, we recorded the blur level attributed to the Sample to match the Target (Matched Blur Level, MBL). Before the experimental session, participants performed a training session (four trials) using stimuli which were not subsequently included in the main experiment. The stimuli and executable files of the experiment are available in the Open Science Framework repository: https://osf.io/d65zs/.

\section{Figure 2}

Illustration of the Experimental Conditions and the Display of Experiment 1 


\section{PRIOR SCENE KNOWLEDGE INCREASES ITS PERCEIVED SHARPNESS}

A.

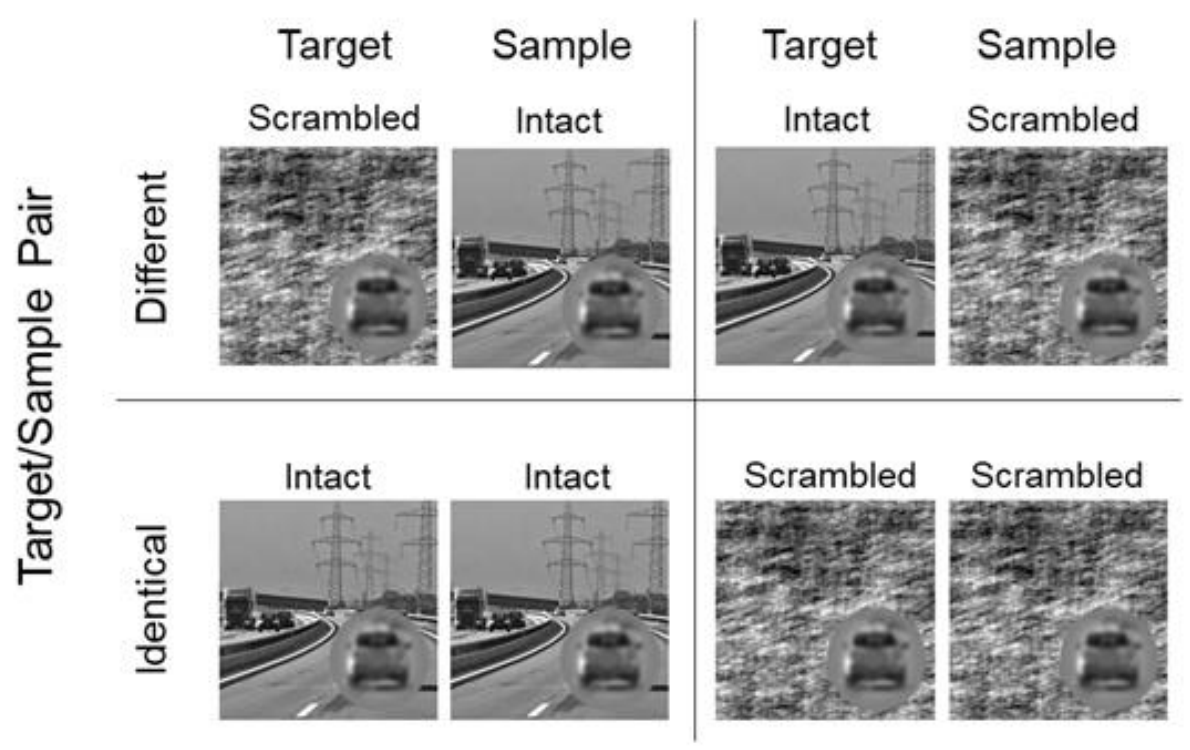

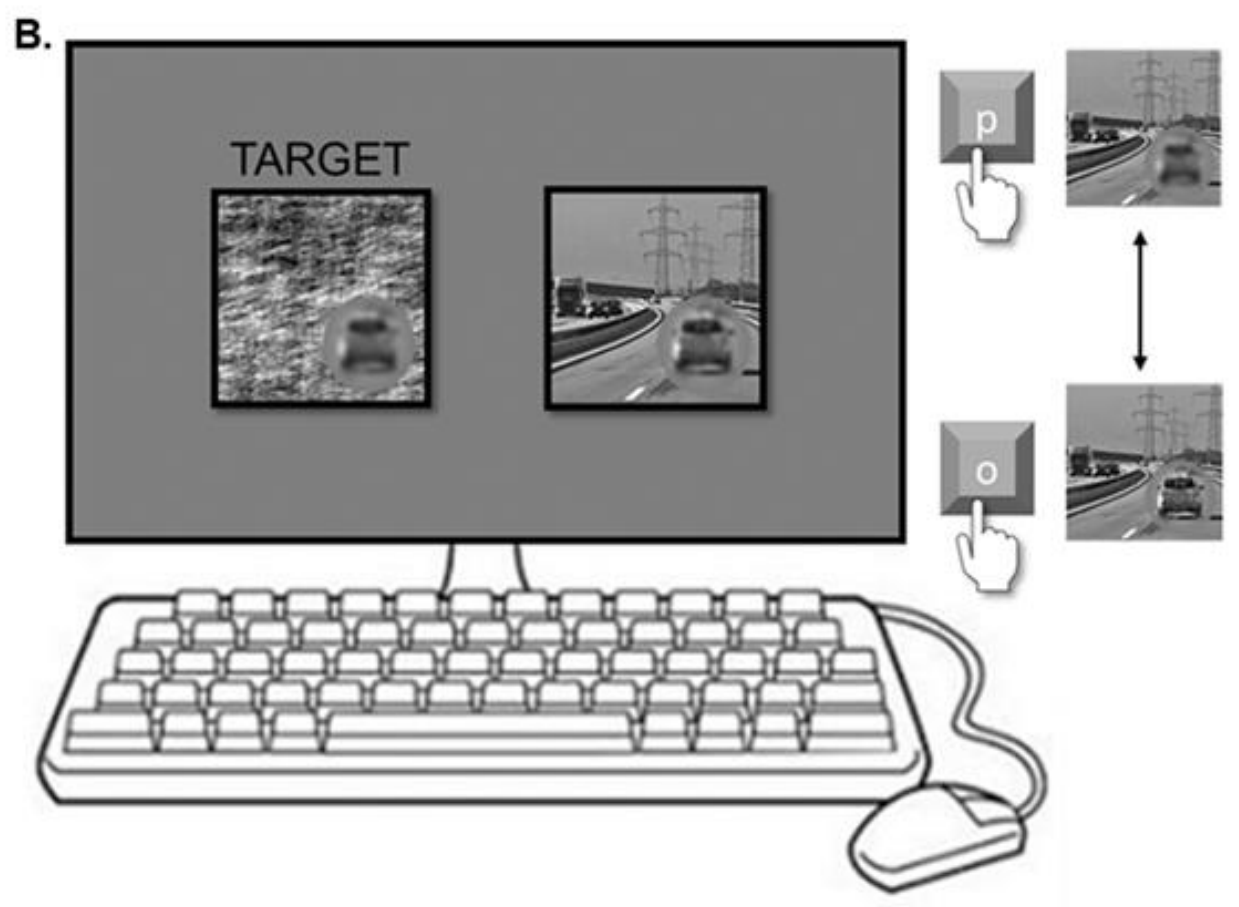

Note. A. Example of stimuli presented in each experimental condition. For each trial, the Target and Sample objects were the same. The backgrounds could be either intact (predictable contextual information) or phase-scrambled (unpredictable contextual information). They either had a different scene background (different Target/Sample pair-types) or the same background (identical Target/Sample pair-types), resulting in four conditions: (1) both the Target and Sample were intact ("T- 
PRIOR SCENE KNOWLEDGE INCREASES ITS PERCEIVED SHARPNESS

intact/S-intact"), (2) both the Target and Sample were scrambled ("T-scramble/S-scrambled"), (3) the Target was intact and the Sample was scrambled ("T-intact/S-scrambled"), and (4) the Target was scrambled and the Sample was intact ("T-scrambled/S-intact"). B. Illustration of the display in a trial with a scrambled Target and an intact Sample (different Target/Sample pair-types). Two scenes containing a blurred object appeared simultaneously, the Target on the left and the Sample on the right. Participants were asked to adjust the Sample object's blur level, by pressing the O and P keys on the keyboard, until they judged both objects as equally blurred.

\section{Data analysis}

Data were analysed using R (R Core Team, 2019) and lme4 package (Bates, Mächler, et al., 2015) to perform a linear mixed effects analysis of the Target/Sample pair-type (identical vs. different) and the Sample's contextual information (intact vs. scrambled) in a $2 \times 2$ withinsubjects design. Contrary to classical analyses of variance (ANOVA; Judd et al., 2012), mixedeffects analyses maximize the generalizability of our results to other participants, but also to other stimuli. We used the Matched Blur Level (MBL) as the dependent variable. Target/Sample pair-type, Sample's contextual information and their interaction term were entered into the model as fixed effects (i.e., as the effects of the variables of interest). Intercepts for subjects and items, as well as subject-wise and item-wise random slopes for the effect of Target/Sample pair-type, Sample's contextual information and their interaction were specified as random effects (i.e., as the effects of the variables to which we want to generalize our results).

We conducted the minimum number of analyses needed to test our hypotheses to avoid increasing the risk of Type-1 error. Based on Lupyan (2017), we expected that at an (objectively) equal level of blur, objects embedded in intact contexts would be subjectively perceived as sharper when compared to objects embedded in scrambled contexts. Therefore, for Target/Sample pairs of different contexts, objects in intact contexts should be blurrier than objects in scrambled contexts to be subjectively perceived as equally blurred. Consequently, 
PRIOR SCENE KNOWLEDGE INCREASES ITS PERCEIVED SHARPNESS

for Target/Sample pairs of different contexts, the MBL of Sample objects in the T-scrambled/Sintact condition should be higher than the MBL of the Sample objects in the T-intact/Sscrambled condition. Alternatively, this effect could be explained by a systematic bias in underestimating the blur of objects embedded in intact contexts irrespective of the context of the Target object. If the difference between MBL of Sample objects in intact and scrambled contexts was due to such a systematic bias, it should be as large regardless of the Target/Sample pair-type (different or identical). On the contrary, a larger difference in MBL for different than identical pair-types would lead to the conclusion that the difference in MBL in the different pair-types is unlikely to be explained by a bias based on the Sample's contextual information. In order to test these hypotheses, we therefore tested the interaction between the contextual information of the Sample and the Target/Sample pair-type, and then the simple effect of contextual information of the Sample object for the Target/Sample pairs of different contexts.

We used the method proposed by Bates, Kliegl, et al. (2015) to construct parsimonious mixed models preventing convergence problems. Visual inspection of residual plots did not reveal any obvious deviations from homoscedasticity or normality. P-values were obtained by Satterthwaite approximation with the lmerTest package (Kuznetsova et al., 2017). The significance threshold was set at .05. Effect sizes were estimated using Cohen's $d_{z}$ (Lakens, 2013). Data and analysis scripts are available in the Open Science Framework repository: https://osf.io/d65zs/.

\section{Results}

Mean MBLs for each experimental condition are shown in Figure 3a. Given that the Target object could be displayed at 20 different blur levels ranging from 6 to 25, a perfect match between the Target and Sample objects would result in a mean MBL of $(6+25) / 2=15.5$. A mean MBL with a value above 15.5 therefore indicates an overestimation of the Sample blur 
PRIOR SCENE KNOWLEDGE INCREASES ITS PERCEIVED SHARPNESS

relative to the Target, while a mean MBL below 15.5 indicates an underestimation of the Sample blur relative to the Target.

Results revealed neither a main effect of the Target/Sample pair-type, $t(18.99)=0.21$, $p=.838, d_{z}=0.037$ (different pair-type: $M=15.40, S E=1.02$; identical pair-type: $M=15.35$, $S E=1)$, nor a main effect of the Sample's contextual information, $t(5078)=1.53, p=.127, d_{z}$ $=0.270$ (intact condition: $M=15.47, S E=1.03$; scrambled condition: $M=15.28, S E=0.99$ ). However, the interaction between the Target/Sample pair-type and Sample's contextual information was significant, $t(5078)=2.06, p=.039, d_{z}=0.364$. In line with our hypotheses, the difference in MBL of Sample objects between intact and scrambled conditions was larger when the Sample and the Target were different (T-scrambled/S-intact: $M=15.63, S E=1.05$; T-intact/S-scrambled: $M=15.17, S E=0.98)$ than when they were identical (T-intact/S-intact: $M=15.31, S E=1 ;$ T-scrambled/S-scrambled: $M=15.38, S E=1)$. Critically, when the Target and the Sample were different, the MBL was significantly higher for a Sample object in the intact than in the scrambled condition, $t(5079.36)=2.53, p=.011, d_{z}=0.448$. Overall, these results support our hypotheses. Participants added more blur to match a Sample object in an intact context to a Target object in a scrambled context than to match a Sample object in a scrambled context to a Target object in an intact context. This suggests that at an objectively equal blur level, objects in intact contexts were subjectively perceived as sharper than objects in scrambled contexts.

Figure $3 \mathrm{~b}$ provides another representation of our results in which the MBL of the Sample object is plotted as a function of the actual blur level of the Target object for the Target/Sample pairs of different contexts only. The dashed line corresponds to a perfect match between the blur of the Sample and Target object. Data points above or below this line indicate an over- or underestimation of the Sample's blur, respectively. Interestingly, visual inspection of this graph suggests that the effect of contextual information on MBL varied according to the 


\section{PRIOR SCENE KNOWLEDGE INCREASES ITS PERCEIVED SHARPNESS}

blur level of the Target object. This effect seems to be null for Target objects with a low blur level below 11 and to be the strongest for Target objects with a high blur level above 16 . Because there was only a limited number of trials per blur level of the Target object (i.e., each Target object was not displayed at all blur levels throughout the experiment, see Methods), the effect of blur level of the Target object was not included as an effect of interest in our model. To get an insight on the significance of this effect, we grouped together trials in which the blur of the Target object was low (below 16) and trials in which the blur of the Target object was high (above 16) and we performed a post-hoc mixed-model analysis to examine whether the effect of contextual information could vary depending on the low vs. high blur level of the Target object. We calculated as a fixed effect the interaction between the Sample's contextual information (intact vs. scrambled) for the Target/Sample pairs of different contexts only and the blur level of the Target object (low vs. high). The interaction was significant, $t(2378.01)=$ $3.15, p=.002, d_{z}=0.557$. This interaction suggests that the Sample's contextual information effect was larger for a high blur level of the Target (above 16; T-scrambled/S-intact: $M=20.98$, $S E=0.57$; T-intact/S-scrambled: $M=20.13, S E=0.55$ ) than a low blur level (below 16 ; $\mathrm{T}$ scrambled/S-intact: $M=10.77, S E=0.61 ;$ T-intact/S-scrambled: $M=10.63, S E=0.56)$.

\section{Figure 3}

Mean Matched Blur Level for Each Condition of Experiment 1 and for the Different Target/Sample Pair-Types According to the Target Object's Blur Level 


\section{PRIOR SCENE KNOWLEDGE INCREASES ITS PERCEIVED SHARPNESS}

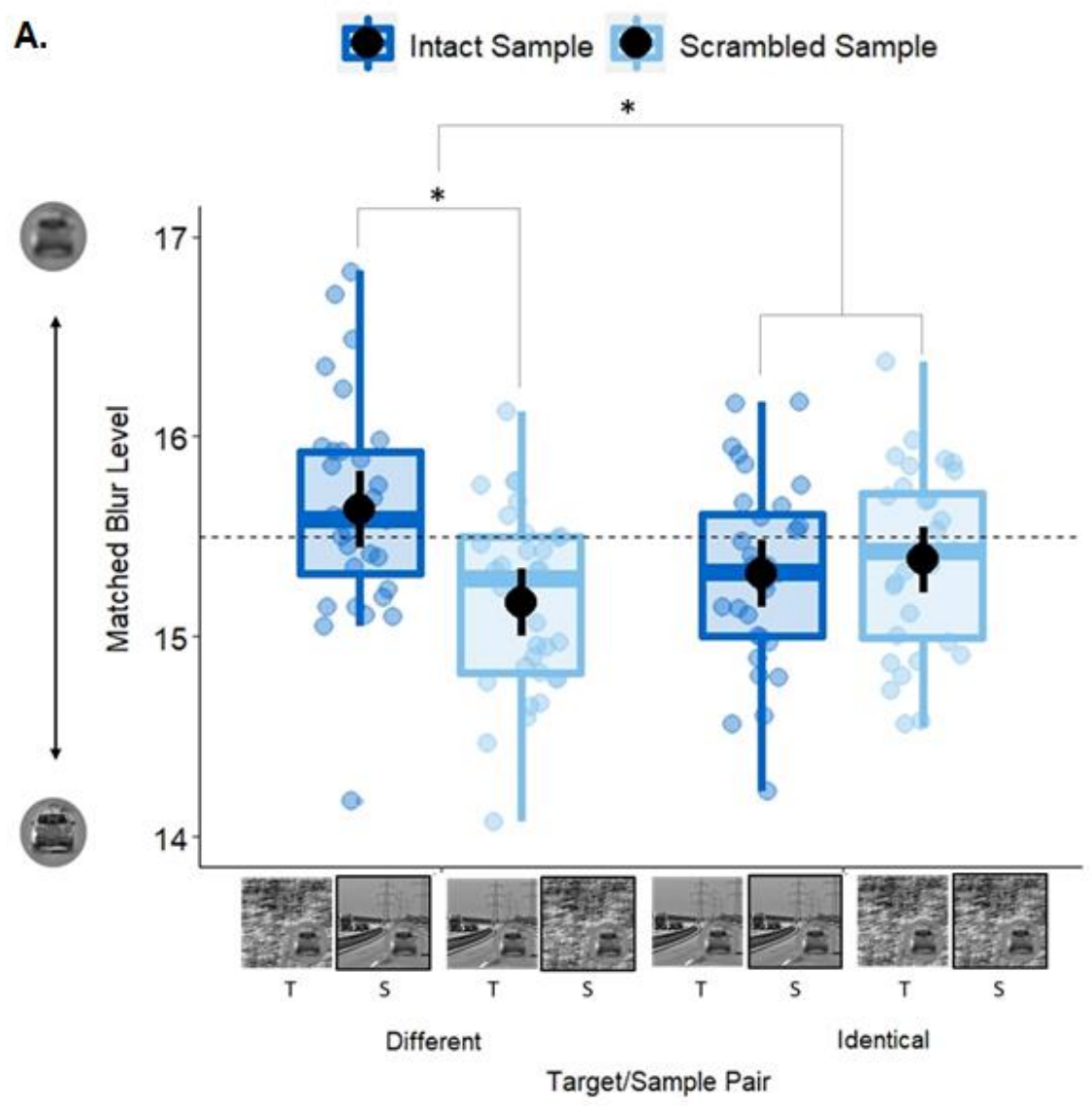

B.

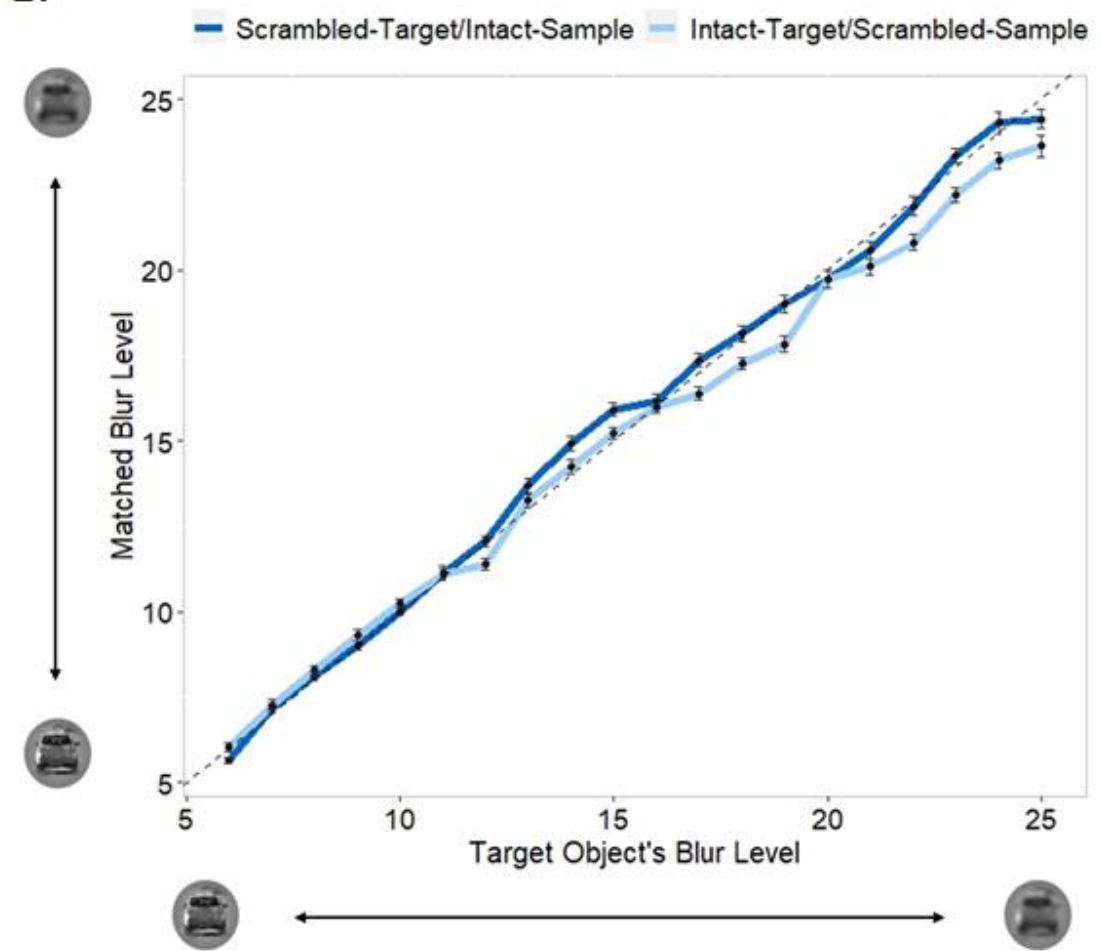

Note. A. Mean matched blur level (MBL) for intact and scrambled Sample object's contextual information according to the Target/Sample pair-type. A high blur level corresponds to a very blurry object whereas a low blur level corresponds to a relatively sharp object. The dashed line represents the 


\section{PRIOR SCENE KNOWLEDGE INCREASES ITS PERCEIVED SHARPNESS}

perfect match between the blur of the Target (T) and Sample (S) objects. A mean matched blur with a value above or below 15.5 indicates an overestimation or underestimation of the Sample's blur relative to the Target, respectively. Black dots and error bars indicate mean and standard error over all individuals and trials, respectively. Color dots correspond to individual observations. B. Mean matched blur level (MBL) for T-scrambled/S-intact and T-intact/S-scrambled conditions according to the Target object's blur level. The dashed line represents the correct Sample object's blur level for each Target object's blur level. Error bars represent standard error over all individuals and trials. $* \mathrm{p}<.05$.

\section{Discussion of Experiment 1}

The purpose of this first experiment was to determine whether expectations based on scene contextual information improves the perceived sharpness of objects. Participants performed a perceptual matching task in which they had to adjust the blur level of an object (Sample) embedded in a predictable intact or an unpredictable scrambled context to match the blur level of the same object (Target) embedded in a predictable intact or an unpredictable scrambled context.

In line with our hypotheses, results showed that when object pairs had a different context (different Target/Sample pair-type), participants matched the blur level of the Target and the Sample objects by attributing to the Sample objects with an intact context (matched to Target objects with a scrambled context) a higher blur level than to the Sample objects with a scrambled context (matched to Target objects with an intact context). This means that at an objectively equal level of blur, objects in an intact context were subjectively perceived as sharper than objects in a scrambled context. To control that this effect of contextual information in Target/Sample pairs with different contexts was not due to (1) a systematic bias in underestimating the blur of the Sample objects embedded in an intact context irrespective of the context of the Target object or (2) a systematic bias in underestimating the blur of the Sample relative to the Target object, we also included in our experiment Target/Sample pair- 


\section{PRIOR SCENE KNOWLEDGE INCREASES ITS PERCEIVED SHARPNESS}

types with an identical context. We observed that the difference between the MBL for a Sample object embedded in an intact context and the MBL for the same Sample object embedded in a scrambled context was actually greater when the Sample and the Target object had a different than an identical context. This indicates that the tendency to underestimate the blur of Sample objects in intact context was larger when they were compared to objects in scrambled context. This therefore argues against an explanation of our results by a systematic bias and rather supports the idea that expectations based on contextual information result in increasing the perceived sharpness of objects. It can also be noted that mean MBL for the identical Target/Sample pairs was below 15.5 suggesting a general tendency to underestimate the level of blur required to match the Sample to the Target objects when they shared a similar context, specifically for very blurry objects with blur level above 16 (see Figure S2a in Supplementary material for MBLs according to the Target blur level in identical pair-types). This could be explained by a response bias in favour of a sharp perception of the Sample during the task whereby participants adapted the Sample to be too sharp than necessary rather than too blurry than necessary. Critically, such a bias strengthens our conclusions in the conditions where Target/Sample pairs were different. Participants added more blur than necessary to Sample objects in intact contexts despite a general tendency to make the Sample sharper than the Target.

Interestingly, post-hoc analyses suggested that the effect of expectations based on contextual information (i.e., effect of contextual information in the different pairs) was significantly larger when the Target object was very blurry (i.e., a blur level above 16) than when it was relatively sharp (i.e., blur level below 16). This result is also in line with predictive coding theories which assume that the influence of expectations on perception increases as signal precision decreases (Friston, 2005; Rao \& Ballard, 1999). 
PRIOR SCENE KNOWLEDGE INCREASES ITS PERCEIVED SHARPNESS

Overall, results of this experiment are consistent with Lupyan's (2017) previous findings that expectations based on prior knowledge increases the perceived sharpness of the visual signal. Importantly, our results suggest that this sharpening mechanism not only applies to reading, which requires processing of details, but also to objects perception. Consequently, we wondered whether these findings could be extended more broadly to the perception of whole scenes that need less details to be identified. This would support the sharpening mechanism as a more general principle of vision. Furthermore, although the object was exactly the same in the Target and the Sample, we cannot exclude that the perceived greater sharpness for objects embedded in intact than scrambled contextual information results from a disruption of object processing in a scrambled than intact context rather than to expectations based on contextual information. Experiment 2 therefore aimed to confirm the present findings and extend them to whole scene perception, while better controlling for signal differences between the Target and Sample stimuli. We used the same perceptual matching task but this time, the Target and Sample stimuli had the exact same signal properties and only differed in terms of orientation: a whole predictable upright scene versus an unpredictable inverted scene.

\section{Experiment 2}

\section{Method}

\section{Participants and Design}

Thirty undergraduate students of Psychology from University Grenoble Alpes (25 women, $\mathrm{M}_{\text {age }}=21.43, \mathrm{SD}_{\text {age }}=4.07$ ) with normal or corrected to normal vision participated in the experiment. This experiment was conducted in parallel to Experiment 1 and the Sample size was estimated similarly as in Experiment 1. It was also performed within the same ethical framework as in Experiment 1.

\section{Stimuli}




\section{PRIOR SCENE KNOWLEDGE INCREASES ITS PERCEIVED SHARPNESS}

The stimuli were constructed from the same 20 photographs of indoor and outdoor scenes $(350 \times 350$ pixels, greyscaled and with an equalized mean luminance of 0.5 as in Experiment 1, using MATLAB R2019b). For each scene, we created two versions in which the scenes were exactly the same but with a different orientation: one in which the scene was left upright (predictable scene layout - upright condition) and the other one in which the scene was vertically flipped around the horizontal axis (unpredictable scene layout - inverted condition). Upright and inverted versions of each scene were then blurred using the same procedure as in Experiment 1 for objects to obtain 31 versions of the image (Figure 4) ranging from a sharp (close to intact) image (blur level $1, \mathrm{SD}=1$ ) to a very blurred and hardly recognizable one (blur level 31, $\mathrm{SD}=10$ ).

\section{Figure 4}

Examples of a Scene at Different Blur Levels Between Blur Levels 1 and 31 in the Upright and

\section{Inverted Conditions}

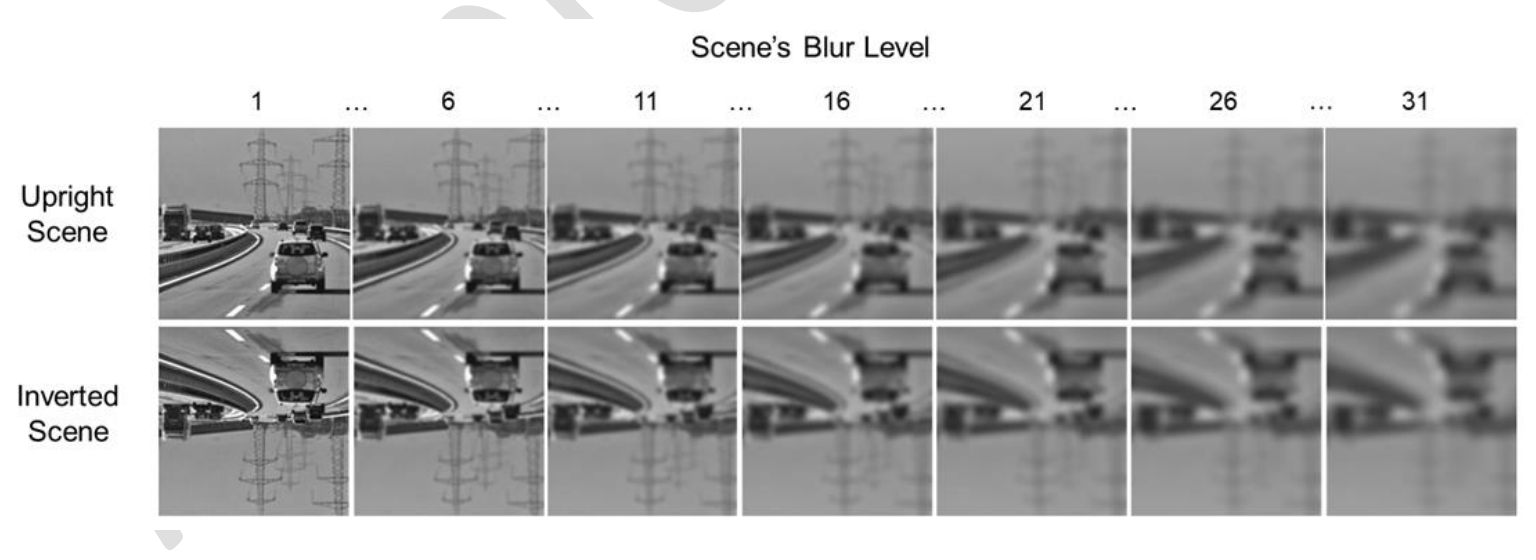

\section{Procedure}

The experimental settings were similar to Experiment 1 (i.e., stimuli displayed using EPrime Go 1.0 software on the participant's personal computer; stimuli and executable files are available in the Open Science Framework repository: https://osf.io/d65zs/). The procedure was 


\section{PRIOR SCENE KNOWLEDGE INCREASES ITS PERCEIVED SHARPNESS}

also similar to Experiment 1 with the difference that the task consisted in matching the blur level of whole scenes instead of objects.

For each trial, the Target and the Sample scenes could be either upright (consistent with prior experience) or inverted (no prior experience). Furthermore, they either had the same (different Target/Sample pair-type) or a different orientation (identical Target/Sample pairtype), resulting in four conditions (see Figure 5a). As in Experiment 1, the scene orientation was not relevant to the task, which consisted in the blur level adjustment. Again, blurring of stimuli had no effect on the mean luminance of stimuli which was set to 0.5 (for pixels intensities between 0 and 1) prior to applying the gaussian filters. Blurring of stimuli only resulted in slightly reducing the RMS contrast of stimuli (from 0.189 at blur level 1 to 0.155 at blur level 31).

An experimental session contained 160 trials appearing in a random order (20 scenes presented twice in each of the four conditions) with a break in approximately the first and second third of the experiment. For all conditions, each of the 20 Target scenes appeared with a blur level varying from 6 to 25 (i.e., a total of 20 blur levels) so that each blur level was presented once per condition for a randomly selected scene. The experiment lasted about 3040 minutes, depending on the speed at which each participant made his/her judgment (mean \pm SD duration of a trial: $2134 \pm 2192 \mathrm{~ms}$ ). For each trial, we recorded the blur level attributed to the Sample to match the Target (Matched Blur Level, MBL). Before the experimental session, participants performed a training session (four trials) using stimuli which were not subsequently included in the main experiment.

\section{Figure 5}

Illustration of the Experimental Conditions and the Display for Experiment 2 


\section{PRIOR SCENE KNOWLEDGE INCREASES ITS PERCEIVED SHARPNESS}

A.

Scene Orientation
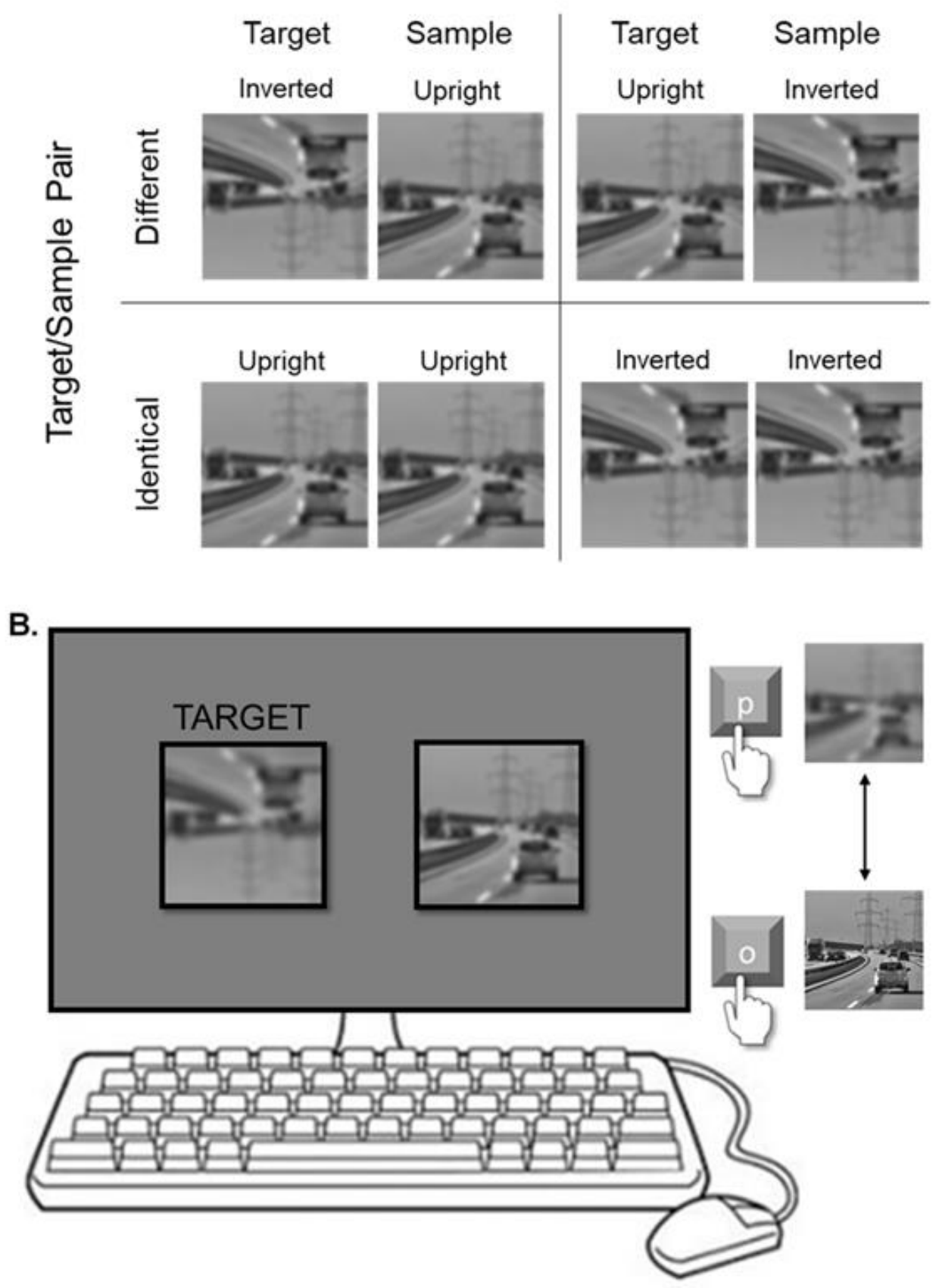

Note. A. Example of stimuli presented in each experimental condition. For each trial, the Target and the Sample scenes could be either upright (consistent with prior experience) or inverted (no prior experience). They either had the same (different Target/Sample pair-type) or a different orientation (identical Target/Sample pair-type), resulting in four conditions: (1) both the Target and Sample scenes were upright, (2) both the Target and Sample scenes were inverted, (3) the Target scene was upright 
and the Sample scene was inverted, and (4) the Target scene was inverted and the Sample scene was upright. B. Illustration of the display in a trial with an inverted Target and an upright Sample (different Target/Sample pair-type). Two blurred scenes appeared simultaneously, the Target on the left and the Sample on the right. Participants were asked to adjust the Sample scene's blur level, by pressing the O and P keys on the keyboard, until they judged both scenes as equally blurred.

\section{Data analysis}

Data analysis and software were similar to Experiment 1 . We performed a linear mixed effects analysis of the Target/Sample pair-type (identical vs. different) and the Sample scene's orientation (upright vs. inverted) in a $2 \times 2$ within-subjects design, with the MBL as a dependent variable. As in Experiment 1, Target/Sample pair-type, Sample scene's orientation, and their interaction term were entered into the model as fixed effects. Intercepts for subjects and items, as well as subject-wise and item-wise random slopes for the effect of Target/Sample pair-type, Sample scene's orientation and their interaction were specified as random effects.

Following the same rationale as in Experiment 1, we tested the interaction between the Sample scene's orientation and the Target/Sample pair-type, as well as the simple effect of the Sample scene's orientation for the Target/Sample pair of different orientations. We used the same method as in Experiment 1 to construct parsimonious mixed models preventing convergence problems and check for deviations from homoscedasticity or normality. P-values were obtained by Satterthwaite approximation with the lmerTest package (Kuznetsova et al., 2017). Data from one participant who did not adjust the blur level of the Sample scene in any trial were discarded from the analysis. Data and analysis scripts are available in the Open Science Framework repository: https://osf.io/d65zs/.

\section{Results}

Mean MBLs for each experimental condition are shown in Figure 6a. As in Experiment 1, a mean MBL of 15.5 corresponds to a perfect match between the Target and Sample scenes 


\section{PRIOR SCENE KNOWLEDGE INCREASES ITS PERCEIVED SHARPNESS}

while a mean MBL with a value above/below 15.5 indicates an over/underestimation of the Sample blur relative to the Target.

Results revealed no main effect of the Target/Sample pair-type, $t(18.99)=0.14, p=$ $.892, d_{z}=0.025$ (different Target/Sample pair-type: $M=15.13, S E=1.05$; identical Target/Sample pair-type: $M=14.97, S E=1.01)$, but we observed a main effect of the Sample scene orientation, $t(4758)=3.63, p<.001, d_{z}=0.662$, with participants attributing a higher MBL to the Sample scene when it was upright $(M=15.28, S E=1.05)$ than inverted $(M=14.83$, $S E=1)$. Moreover, the interaction between the Target/Sample pair-type and the Sample scene orientation was significant, $t(4758)=3.55, p<.001, d_{z}=0.649$. In line with our hypotheses, the difference in MBL of Sample scenes between upright and inverted conditions was larger when the Sample and the Target scenes had a different orientation (T-inverted/S-upright: $M=$ $15.58, S E=1.07 ;$ T-upright $/ \mathrm{S}$-inverted: $M=14.68, S E=1.01)$ than an identical orientation $(\mathrm{T}$ upright/S-upright: $M=14.98, S E=1.02$; T-inverted/S-inverted: $M=14.97, S E=0.99$ ). Critically, when the Target and the Sample scenes had a different orientation, the MBL was significantly higher for a Sample scene in the upright than in the inverted condition, $t(4758)=$ 5.08, $p<.001, d_{\mathrm{z}}=0.927$. Overall, these results support our hypotheses. Participants added more blur to match an upright Sample scene to an inverted Target scene than to match an inverted Sample scene to an upright Target scene.

As in Experiment 1, Figure 6b provides another representation of our results in which the MBL of the Sample scene is plotted as a function of the blur level of the Target scene for Target/Sample pairs of different orientations only. The dashed line corresponds to a perfect match between the blur of the Sample and Target scene and data points above or below this line indicate an over- or underestimation of the Sample's blur, respectively. Once again, visual inspection of this graph suggests that the effect of the Sample scene orientation on MBL varied according to the blur level of the Target scene. As in Experiment 1, we calculated in a post-hoc 
PRIOR SCENE KNOWLEDGE INCREASES ITS PERCEIVED SHARPNESS

analysis the interaction between the Sample scene's orientation for Target/Sample pairs of different orientations only and the blur level of the Target scene (low vs. high). However, the interaction was not significant, $t(2227.56)=1.86, p=.063, d_{z}=0.339$, suggesting that the Sample scene's orientation effect was not significantly larger for a high blur level of the Target (above 16; T-inverted/S-upright: $M=20.86, S E=0.59$; T-upright/S-inverted: $M=19.74, S E=$ 0.56 ) than a low blur level (below 16 ; T-inverted/S-upright: $M=10.80, S E=0.62$; T-upright/Sinverted: $M=10.12, S E=0.54)$.

\section{Figure 6}

Mean Matched Blur Level for Each Condition of Experiment 2 and for Different Target/Sample Pair-Types According to the Target Scene's Blur Level 


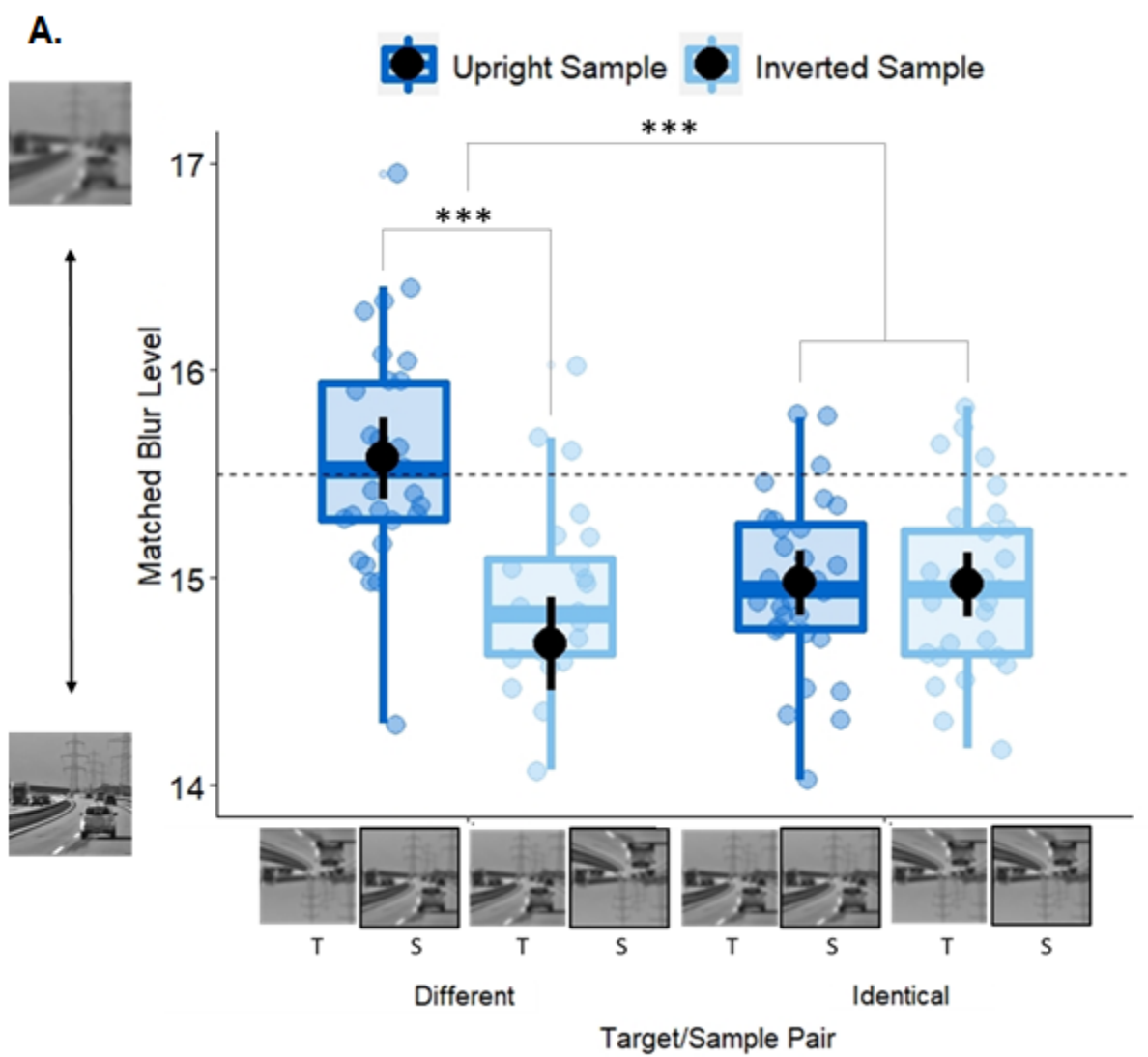

B.

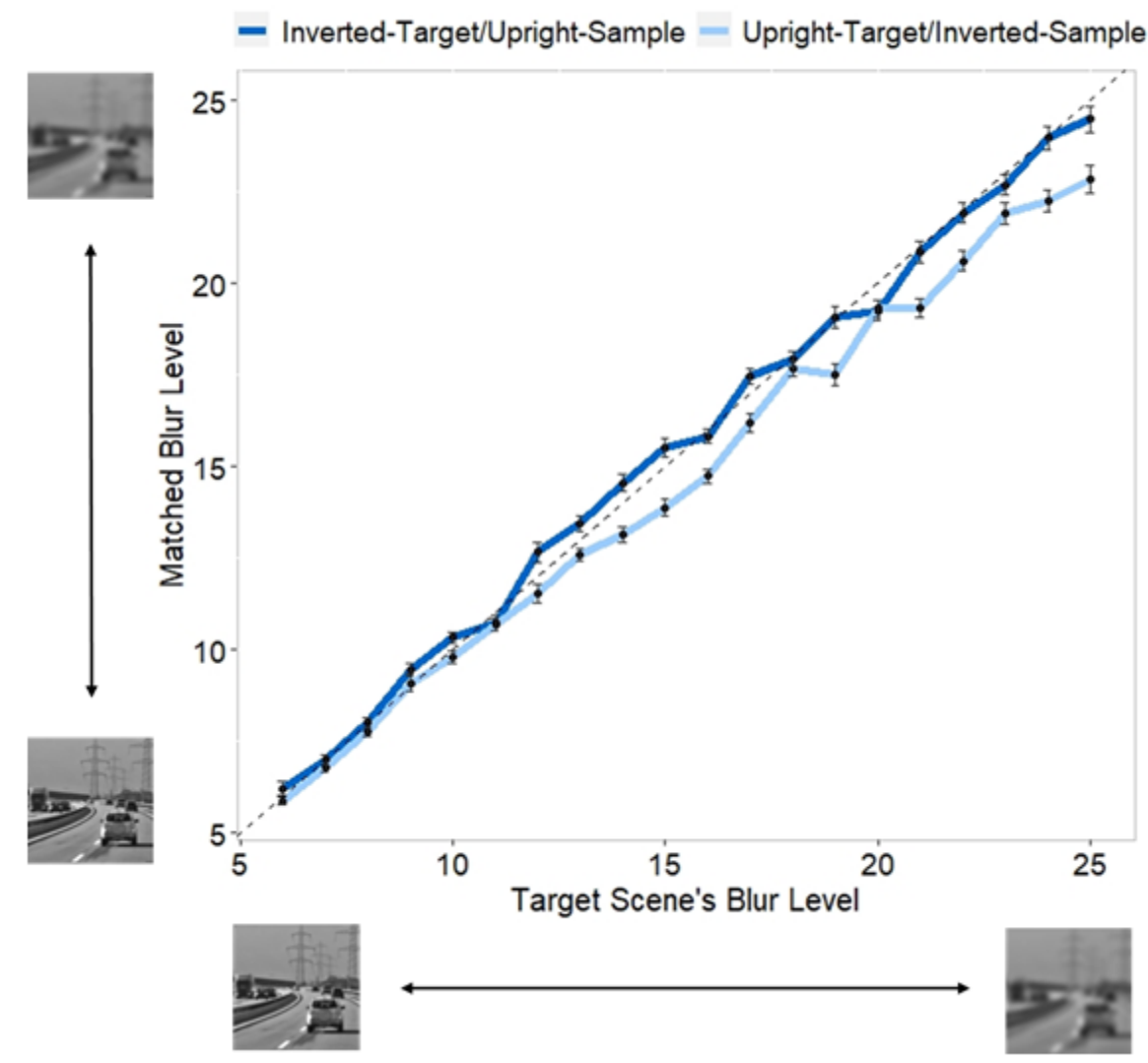




\section{PRIOR SCENE KNOWLEDGE INCREASES ITS PERCEIVED SHARPNESS}

Note. A. Mean matched blur level (MBL) for upright and inverted Sample scenes according to the Target/Sample pair-type. A high blur level corresponds to a very blurry scene whereas a low blur level corresponds to a relatively sharp scene. The dashed line represents the perfect match between the blur of the Target (T) and Sample (S) scenes. A mean matched blur with a value above or below 15.5 indicates an overestimation or underestimation of the Sample's blur relative to the Target, respectively. Black dots and error bars indicate mean and standard error over all individuals and trials, respectively. Color dots correspond to individual observations. B. Mean matched blur level (MBL) for T-inverted/Supright and T-upright/S-inverted conditions according to the Target scene's blur level. The dashed line represents the correct Sample scene's blur level for each Target scene's blur level. Error bars represent standard error over all individuals and trials.

$* * * \mathrm{p}<.001$

\section{Discussion of Experiment 2}

The purpose of this second experiment was to determine whether expectations based on prior experience increase the perceived sharpness of whole scenes. It also allowed us to ensure that the results from Experiment 1 could not be explained by visual signal differences between the Target and Sample images. We used the same perceptual matching task as in Experiment 1, in which participants had to adjust the blur level of a predictable upright or unpredictable inverted scene (Sample) to match the blur level of the same predictable upright or unpredictable inverted scene (Target).

We replicated the results of Experiment 1 by showing that when Target/Sample pairs had a different orientation (different Target/Sample pair-types), participants matched the blur level of the Target and the Sample scenes by attributing to the upright Sample scene a higher blur level than to the inverted Sample scene. This means that at an objectively equal level of blur, upright scenes would be subjectively perceived as sharper than inverted ones. As in Experiment 1, the significant interaction between the Sample scene orientation and the Target/Sample pair-type suggested that the effect of Sample scene orientation was larger in 


\section{PRIOR SCENE KNOWLEDGE INCREASES ITS PERCEIVED SHARPNESS}

different than identical pair-types, allowing to rule out systematic biases (tendency to underestimate the blur of the Sample relative to the Target; tendency to underestimate the blur of upright scenes irrespective of what they are being matched to) which could explain our results when the Target and the Sample had a different orientation. Moreover, as in Experiment 1, participants had a general tendency to underestimate the level of blur required to match the Sample to the Target scenes when they shared a similar context, specifically for very blurry scenes with a blur level above 16 (see Figure S2b in Supplementary material for MBLs according to the Target blur level in identical pair-types). Overall, results of this experiment confirm results of Experiment 1. Importantly, these results further allow us to extend these findings to whole scene perception suggesting that the sharpening mechanism is a more general principle of vision which applies to many stimuli irrespective of the level of details they require to be identified.

The post-hoc analysis on the effect of the Target blur level failed to replicate results of Experiment 1. Although the scene orientation effect when the Target and the Sample were different tended to increase with the blur level of the Target, this difference did not reach significance in the present experiment. As in experiment 1, these exploratory analyses were performed to refine our interpretation of the results. However, Experiments 1 and 2 were not designed to test this hypothesis. In particular, in order to reduce the amount of trials and the duration of both experiments, each of the 20 Target stimuli used was only presented twice per condition as the Target, and they were associated with only two different blur levels. Thus, the blur level of the Target and the Target stimulus identity were not independent (i.e., a Target stimulus seen with a high level of blur was not necessarily seen with a low level of blur). Therefore, we cannot ensure that the observed tendencies are due to the signal precision (i.e., Target blur level) and not to stimulus identity. For this reason we conducted a control experiment (see Experiment 2bis in the Supplementary material) which replicated Experiment 


\section{PRIOR SCENE KNOWLEDGE INCREASES ITS PERCEIVED SHARPNESS}

2 for the different Target/Sample pair-types in which all Target stimuli could be seen at four blur levels (ranging from low to high), so that the Target blur level was manipulated independently of the stimulus identity. The results of Experiment 2bis supported post-hoc analyses of Experiments 1 and 2 by showing that the effect of expectations increased as the signal precision decreased. However, it increased up to a certain blur threshold where the influence of expectations on the perceived sharpness remained constant although the signal precision continued to decrease.

The perceptual matching task used in Experiments 1 and 2 allowed us to implicitly assess the relative perceived sharpness of predictable and unpredictable stimuli. The fact that participants made predictable stimuli blurrier than unpredictable ones to judge both stimuli as equally blurred led us to the assumption that at an equal level of blur, predictable stimuli were perceived as sharper than unpredictable ones. However, this was not explicitly tested in our experiments. We therefore conducted a third experiment in order to confirm results of Experiments 1 and 2 using a task involving explicit judgments on the sharpness of visual stimuli. We presented participants with pairs of upright and inverted scenes used in Experiment 2 but this time participants were required to indicate whether they perceived a blur difference between the two scenes and if so, which of the two scenes was perceived as the blurriest or as the sharpest. Critically, the two scenes had the same objective blur level in most trials. In line with results of Experiments 1 and 2, we expected participants to explicitly judge upright scenes as sharper than inverted scenes in these trials. In this experiment, we additionally recorded participants' eye movements to assess the extent to which predictable and unpredictable stimuli elicited differential amounts of visual attention as indexed by fixation duration. Indeed, visual attention has been found to enhance visual perception, for example resulting in increased perceived contrast or spatial resolution (Carrasco et al., 2004; Yeshurun \& Carrasco, 1998). It 
is thus possible that increased perceived sharpness for predictable stimuli can be explained by the fact that predictable stimuli elicited more visual attention than unpredictable ones.

\section{Experiment 3}

\section{Method}

\section{Participants and Design}

Thirty-five undergraduate students of Psychology from University Grenoble Alpes (31 women, $\left.\mathrm{M}_{\mathrm{age}}=19.63, \mathrm{SD}_{\mathrm{age}}=2.06\right)$ with normal or corrected to normal vision participated in the experiment. The sample size was set based on an estimated effect size of $d_{z}=0.93$ from the observed effect of predictability in the different pair condition from Experiment 2 to achieve a power (i.e., 1 - $\beta$, with $\beta$ being the risk of Type- 2 error) of 0.8 with an alpha level of 0.025 after Bonferroni correction for the two planned non-orthogonal analyses on test trials. The present experiment was performed within the same ethical framework as in Experiments 1 and 2.

\section{Stimuli and Procedure}

The stimuli were exactly the same blurred upright and inverted scenes as those used in Experiment 2. Contrary to Experiments 1 and 2, this experiment was conducted after the Covid19 lockdown periods in a laboratory allowing us to couple our behavioral measures to eye movement recordings. The experiment was thus constructed using the Psychtoolbox (Brainard, 1997; Pelli, 1997) implemented in MATLAB R2019b (MathWorks, Natick, MA, USA). Stimuli were displayed on a 30' monitor (DELL ULTRASHARP) with a resolution of $1920 \times$ 1080 pixels (experimental script available in the Open Science Framework repository: https://osf.io/d65zs/). Participants put their head on a chin rest $70 \mathrm{~cm}$ away from the screen to keep their head fixed for the recording of eye movements. Eye movements were recorded using an Eyelink 1000 eye-tracker (SR Research, Ontario, Canada) with a sampling rate of 1,000 Hz 


\section{PRIOR SCENE KNOWLEDGE INCREASES ITS PERCEIVED SHARPNESS}

and a nominal spatial resolution of $0.01^{\circ}$ of visual angle. For each participant, we recorded only the left eye using the "pupil-corneal reflection" mode. The Eyelink software automatically detected saccades with the following thresholds: speed $>30 \%$, acceleration $>8,000^{\circ} / \mathrm{s}^{2}$, and saccadic displacement $>0.15^{\circ}$. Fixations were detected when the pupil was visible and no saccade was in progress. Blinks were detected during partial or total occlusion of the pupil. Before starting the experiment and every 50 trials, we set up a calibration procedure in which participants had to orient their gaze toward five separate dots appearing sequentially at the centre and at each edge of the screen. A drift correction was performed every 10 trials.

In this experiment, a trial began with a fixation cross appearing at the centre of the screen. Then two versions of the same scene were displayed on the left and right of the screen on a grey background (average luminance of 0.5 ) on each trial. The experimental session contained two types of trials: "test" and "catch" trials. In test trials (Figure 7a), the two scenes had the same blur level which could be of 5, 15, or 25 (i.e., low, medium, and high blur level). In this experiment, we only used three blur levels in order to ensure that, contrary to Experiments 1 and 2, all stimuli would be seen at the same blur levels in each experimental condition while keeping the experiment relatively short. For test trials, the two scenes always had a different orientation (one upright predictable scene and one inverted unpredictable scene). The upright scene was displayed on the right and the inverted scene on the left on half of the test trials and the opposite was done in the other half. Participants were told that the blur difference between the scenes could be subtle and were instructed to find which of the two scenes was the blurriest or the sharpest (Figure 7c). It is possible that when participants have doubts about whether there is a difference in blur between the two scenes, they could be prone to systematically select the more familiar (the upright scene) one (Han \& VanRullen, 2016). To cancel out any effect of such response bias, half of the participants were instructed to report the scene they judged as the blurriest while the other half had to report the scene they judged 


\section{PRIOR SCENE KNOWLEDGE INCREASES ITS PERCEIVED SHARPNESS}

as the sharpest. They also had the option to answer that they did not perceive any blur difference between the two scenes. Test trials were the trials of interest allowing us to test our hypothesis that at an objectively equal level of blur, participants would perceive inverted scenes as blurrier than upright scenes. We also included catch trials (Figure $7 b$ ) to ensure that participants performed the task properly when the blur of the two scenes was objectively different and to prevent them from realising that both scenes had often the same blur level. In these catch trials, the two scenes had different blur levels. One of the two scenes had a blur level of 5, 15, or 25 . The other one had a blur of 3 to 7 levels above or below. The blurriest scene was on the right for half of the catch trials and on the left for the other half. Furthermore, for catch trials, the two scenes either had a different orientation (i.e., one upright predictable scene and one inverted unpredictable scene) or an identical orientation (i.e., both scenes were upright or inverted). Catch trials in which the two scenes had an identical orientation were added in order to prevent participants from adopting a strategy consisting of systematically selecting the upright or inverted scene. Given that Experiment 2 revealed that participants made upright scenes blurrier (by up to 1.73 blur levels on average) than inverted scenes to perceive both scenes as equally blurred, the minimal difference between the blur levels of the two scenes in catch trials was set to 3 in order to ensure that the two scenes would be perceived as having a different blur level. Participants had to give their response (Figure 7c) by pressing the "UpArrow" key if they did not perceive a difference between the two scenes, the "LeftArrow" key if they judged the left scene as the blurriest (or as the sharpest) and the "RightArrow" key if they judged the right scene as the blurriest (or as the sharpest).

\section{Figure 7}

Illustration of the Experimental Conditions and the Display for Experiment 3 


\section{PRIOR SCENE KNOWLEDGE INCREASES ITS PERCEIVED SHARPNESS}

A.

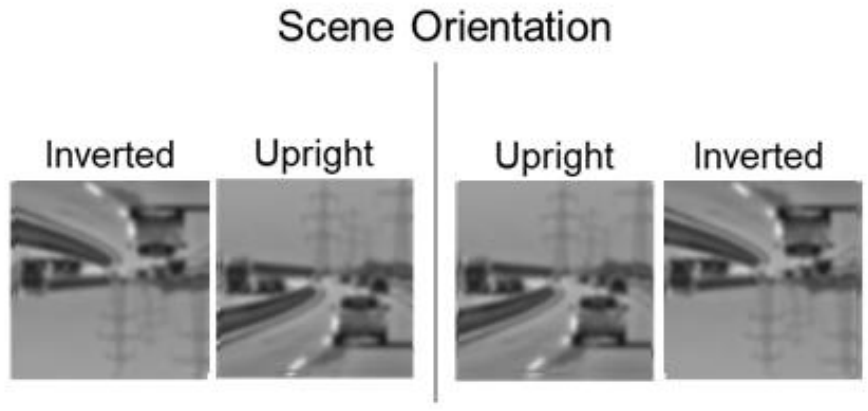

B.

\section{Scene Orientation}

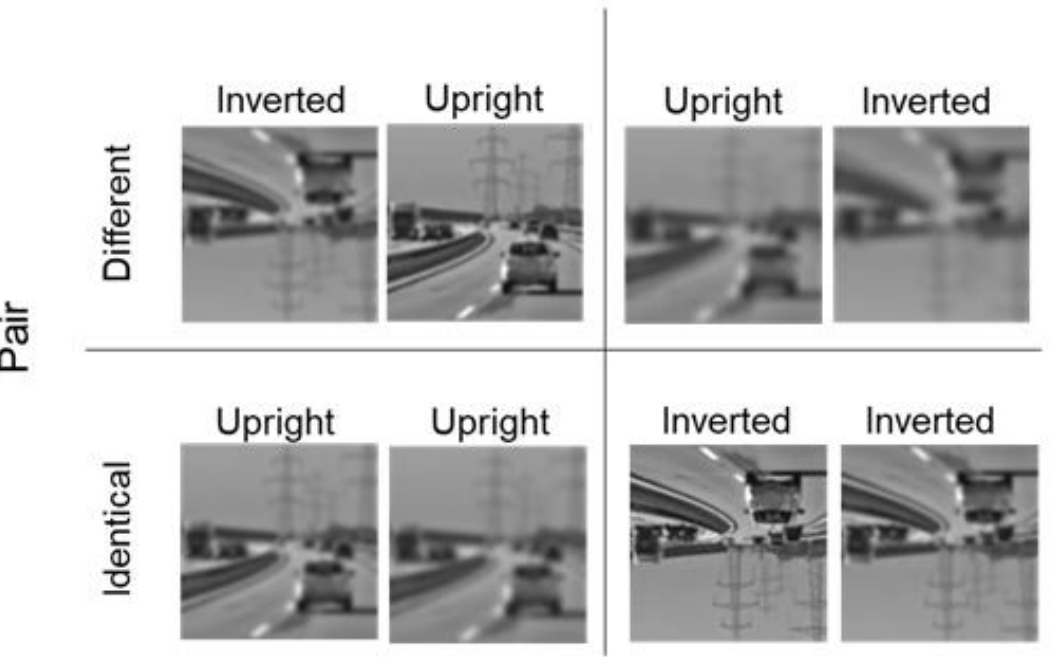

c.

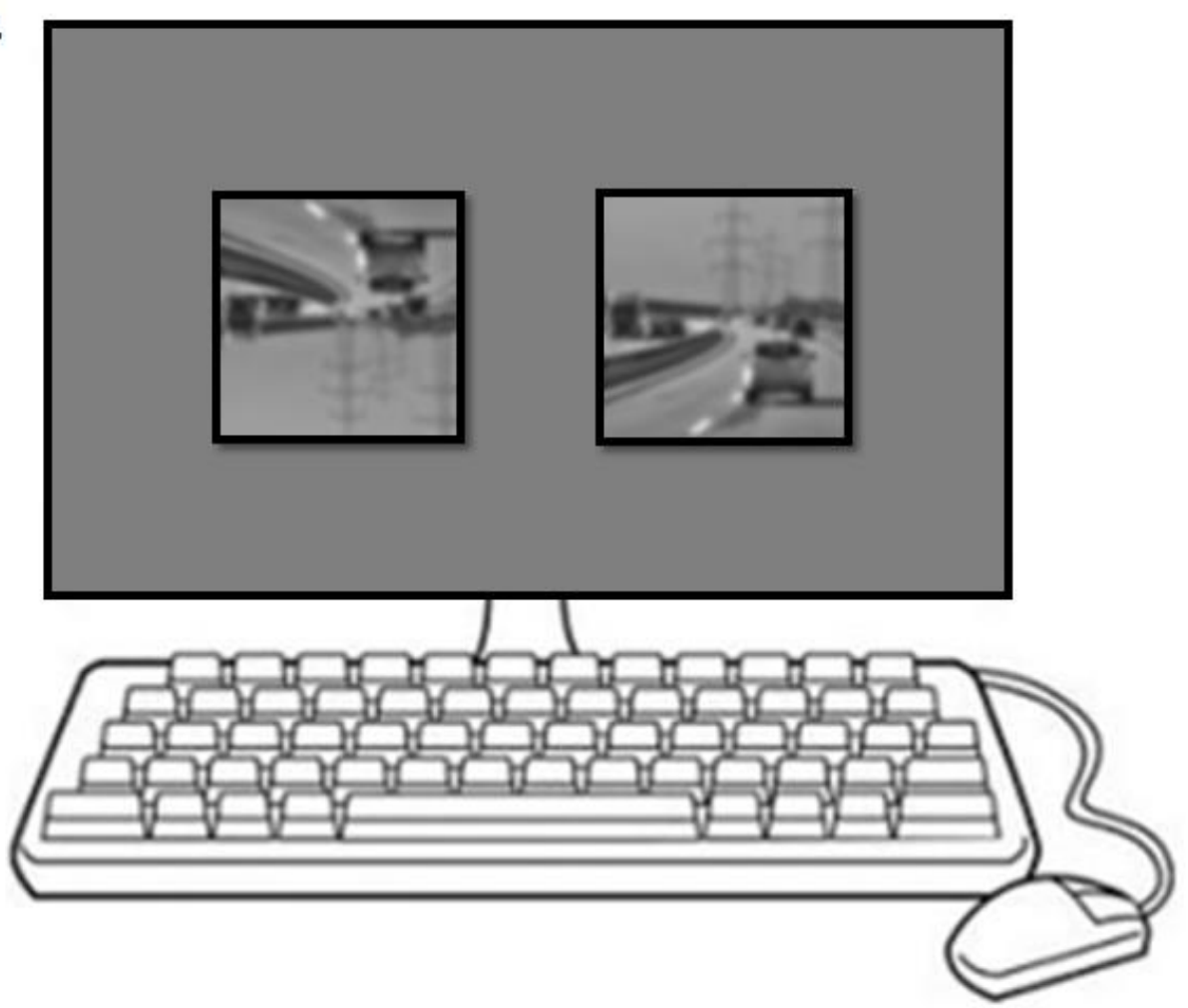




\section{PRIOR SCENE KNOWLEDGE INCREASES ITS PERCEIVED SHARPNESS}

Note. A. Example of stimuli presented in test trials. Both scenes of a pair always had a different orientation (one upright and one inverted scene), but the same blur level. B. Example of stimuli presented in test trials. Both scenes of a pair had either the same or a different orientation, and they had different blur levels. C. Illustration of the display. Two upright or inverted blurred scenes appeared simultaneously either with the same (test trials) or with different (catch trials) blur levels. Participants were asked to find which of the two scenes was the blurriest or the sharpest depending on their experimental group.

The experimental session contained 120 test trials $(20$ scenes pairs $\times 2$ orientation combinations [left scene upright - right scene inverted vs. left scene inverted - right scene upright] $\times 3$ blur levels [5 vs. 15 vs. 25]) and 80 catch trials $(20$ scenes pairs $\times 2$ pairs [both scenes with identical vs. different orientations] $\times 2$ orientations [upright scene vs. inverted scene]). This resulted in a total of 200 randomly displayed trials with a break every 50 trials. For each trial, we recorded whether participants perceived a blur difference between the scenes and if so which scene they chose as the blurriest or as the sharpest depending on the experimental group. We also recorded the fixation durations on each scene. The experiment lasted about 25 minutes, depending on the speed at which participants made their judgment (mean \pm SD duration of a trial: $3124 \pm 2924 \mathrm{~ms}$ ).

\section{Data analysis}

\section{Behavioral data}

Data were analysed using the same software and package as in the previous experiments. To analyse test trials (i.e., on which both scenes had the same blur level), we set up two mixed effects logistic regression models. The first logistic regression aimed to evaluate whether or not participants perceived a blur difference between the two images of test trials (i.e., the dependent variable was perceived difference vs. no perceived difference). The second logistic regression allowed us to evaluate whether participants judged more often the 


\section{PRIOR SCENE KNOWLEDGE INCREASES ITS PERCEIVED SHARPNESS}

predictable or unpredictable scene as the blurriest in test trials (i.e., the dependent variable was predictable scene judged as the blurriest vs. unpredictable scene judged as the blurriest). For both logistic regressions, the intercept and the blur level of the scenes were entered as fixed effects. Intercepts for subjects and items, as well as subject-wise and item-wise random slopes for the effect of the blur level were specified as random effects. Based on results from Experiment 2, we expected that at an objectively equal level of blur, upright scenes would be explicitly perceived as sharper when compared to inverted scenes. To test this hypothesis, we tested 1) if the proportion of perceived blur difference in test trials was significantly greater than chance and 2) if the proportion of inverted scenes judged as the blurriest was significantly greater than chance. As in Experiment 2, we also tested whether this effect was modulated by the signal precision (i.e., blur level) of the scenes in test trials. If the influence of expectations on perceived sharpness increases as the signal precision decreases, we expected the tendency to select the inverted scene as the blurriest to increase with increasing blur level of scene pairs (i.e., interaction between the effect of predictability and blur level). To test this hypothesis, we calculated the effect of the blur level on the proportion of inverted scenes judged as the blurriest and we also performed pairwise comparisons between each blur level.

To analyse catch trials (i.e., when the two scenes had objectively different blur levels), we also set up a mixed effects logistic regression model with the accuracy in detecting blur differences as a dependent variable. Pair-type (i.e., both scenes having or not the same orientation) was entered into the model as fixed effect. Intercepts for subjects and items, as well as subject-wise and item-wise random slopes for the effect of pair-type were entered as random effects. Based on this model, we calculated whether the accuracy in each condition differed significantly from chance to ensure that participants perceived the blur level difference between the two images and performed the task properly. 
PRIOR SCENE KNOWLEDGE INCREASES ITS PERCEIVED SHARPNESS

For all analyses, the significance of the effects was tested with a Wald test. The significance threshold was set at 0.025 for main effects of blur level on test trials after Bonferroni correction for two planned non-orthogonal logistic regressions $(.05 / 2=.025)$, at 017 for three a posteriori pairwise comparisons on test trials analyses after Bonferroni correction for three non-orthogonal tests $(.05 / 3=.017)$ and at .017 for catch trials analyses after Bonferroni correction for three non-orthogonal tests on the accuracy. Data and analysis scripts are available in the Open Science Framework repository: https://osf.io/d65zs/.

\section{Eye movements data}

For eye movements analyses, we defined for each trial two areas or interest (AOI) corresponding to the location of the two scenes on the screen. We then extracted the total fixation duration in each of these AOIs for each trial. We ran eye movements analyses on test trials only, to compare the fixation durations as an index of visual attention between the upright and inverted scenes when both scenes had an objectively equal blur level and were differently oriented. This was done to test whether increased perceived sharpness of upright relative to inverted scenes in test trials could be explained by increased visual attention to upright relative to inverted scenes, as visual attention has been shown to enhance visual perception(Yeshurun \& Carrasco, 1998). We performed a linear mixed effects analysis of the Scene orientation (upright vs. inverted) with the total fixation duration of the scene as the dependent variable. Intercept and Scene orientation terms were entered into the model as fixed effects. Intercepts for subjects and items, as well as subject-wise and item-wise random slopes for the effect of Scene orientation were specified as random effects. We calculated the main effect of Scene orientation (upright vs. inverted) on fixation duration to assess differences in visual attention between upright and inverted scenes. 
PRIOR SCENE KNOWLEDGE INCREASES ITS PERCEIVED SHARPNESS

Mixed models were constructed using the same method as in Experiments 1 and 2 (i.e., using Bates, Kliegl, et al., 2015's method for building parsimonious models). Data and analysis scripts are available in the Open Science Framework repository: https://osf.io/d65zs/.

\section{Results}

\section{Behavioral results}

Mean proportions of responses for each blur level of test trials are shown in Figure 8a. Mean accuracy for each experimental condition of catch trials is shown in Figure 8b. Eight participants were removed from the analyses due to an accuracy equal or lower than chance in catch trials, indicating that they did not get involved in the task (in fact, these participants did not perform the task and systematically reported "no difference"), resulting in a final sample of 27 participants.

First, the analyses conducted on catch trials revealed that when the two scenes appeared with an objectively different blur level, the accuracy in selecting the blurriest one was high for each condition (different pair-type: $M=0.87, S E=0.07$; identical pair-type: $M=0.89, S E=$ 0.06 ) and significantly above chance (different pair-type: $\beta=2.37, z=7.92, p<.001$; identical pair-type: $\beta=2.58, z=8.57, p<.001$ ). Although the minimal difference between the blur levels of the two scenes was set to 3 to ensure that the two scenes were perceived as having a different blur level, we wondered whether the accuracy decreased when the upright scene was objectively blurrier than the inverted scene relative to the reverse. According to our hypotheses, the upright scene should be perceived as sharper than the inverted one. However, when the upright scene was objectively blurrier than the inverted scene, the increased perceived sharpness of the upright one may have compensated for the difference in blur levels between the two scenes, resulting in a reduced perceived difference. To this end, we performed a mixed effects logistic regression on the catch trials where the two scenes were differently oriented. 


\section{PRIOR SCENE KNOWLEDGE INCREASES ITS PERCEIVED SHARPNESS}

The intercept and the orientation of the objectively blurriest scene (i.e., upright or inverted) were entered as fixed effects. Intercepts and random slopes for the effect of the blurriest scene orientation were specified as random effects for the subjects and stimuli. The results showed that the accuracy was significantly lower when the upright scene was blurrier than the inverted one $(M=0.80, S E=0.08)$ relative to when the inverted scene was blurrier than the upright one $(M=0.94, S E=0.05), \beta=2.28, z=5.45, p<.001$, arguing in favor of the sharpening mechanism during scene perception.

Regarding test trials, results showed that participants perceived a blur difference $(M=$ $0.61, S E=0.09)$ significantly more frequently than no blur difference $(M=0.39, S E=0.09)$ even though both scenes had the same objective blur level, $\beta=0.68, z=2.60, p=.009$, supporting an influence of predictability on blur perception. Moreover, when they perceived a blur difference, participants chose the inverted scene as the blurriest $(M=0.79, S E=0.08)$ more frequently than the upright scene $(M=0.21, S E=0.08), \beta=1.42, z=11.19, p<.001$. Thus, participants perceived upright scenes as sharper than inverted scenes at an objectively equal level of blur. However, contrary to what was expected, the proportion of inverted scene choice as the blurriest decreased as the blur level of the scene pairs increased (for a low blur of 5: $M=0.62, S E=0.09$; for a medium blur of $15: M=0.63, S E=0.09$; for a high blur of 25: $M$ $=0.57, S E=0.10), \beta=-0.02, z=-2.32, p=.020$. After Bonferroni correction, pairwise comparisons failed to demonstrate a significant difference on the proportion of inverted scene choice as the blurriest between the different blur levels, neither from a blur level of 5 to $15, \beta$ $=0.29, z=1.75, p=.081$, nor from a blur level of 15 to $25, \beta=0.17, z=1.13, p=.257$, nor from a blur level of 5 to $25, \beta=0.47, z=2.32, p=.020$.

\section{Figure 8}




\section{PRIOR SCENE KNOWLEDGE INCREASES ITS PERCEIVED SHARPNESS}

Mean Proportion of Scenes Judged as the Blurriest According to the Blur Level for Test Trials and Mean Accuracy According to the Type of Pair and the Orientation of the Objectively Blurriest Scene for Catch Trials of Experiment 3.

A. Inverted Upright $\stackrel{t}{+}$ No Difference

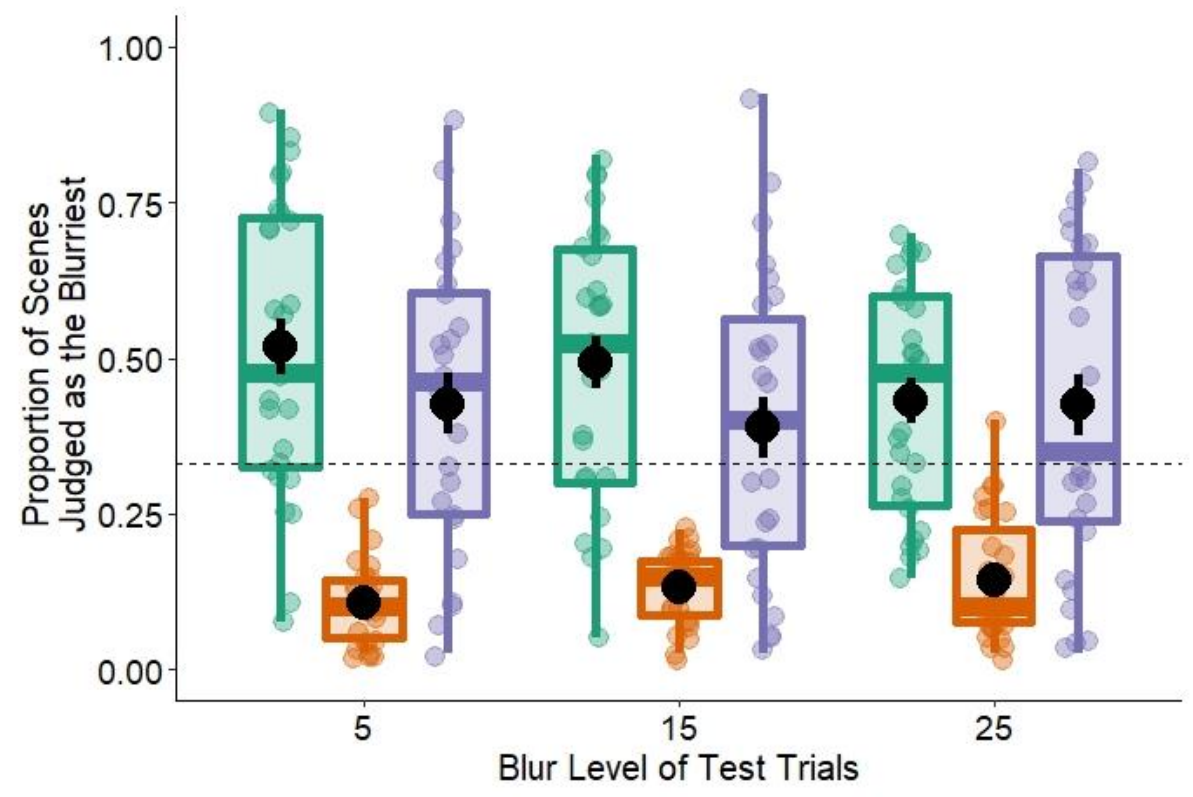

B. Upright objectively blurrier Inverted objectively blurrier

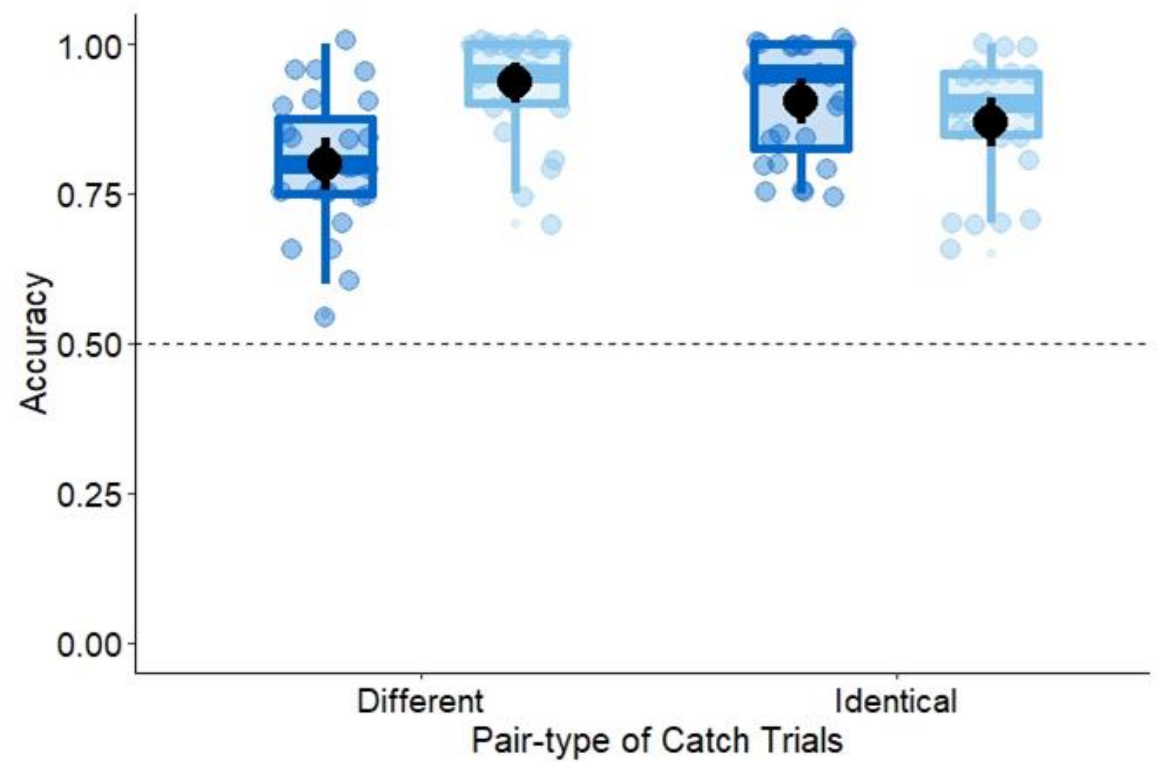

Note. A. Mean proportion of scenes judged as the blurriest according to the blur level for the test trials (i.e., trials in which both scenes have the same objective blur level). The two scenes in test trials always 


\section{PRIOR SCENE KNOWLEDGE INCREASES ITS PERCEIVED SHARPNESS}

had a different orientation. Black dots and error bars indicate mean and standard error over all individuals and trials, respectively. Color dots correspond to individual observations. The dashed line represents chance. B. Mean accuracy for the catch trials (i.e., trials in which the two scenes have an objectively different blur level) according to the orientation of the objectively blurriest scene and the type of pair. The two scenes in catch trials either had a different orientation or an identical orientation. Black dots and error bars indicate mean and standard error over all individuals and trials, respectively. Color dots correspond to individual observations. The dashed line represents chance.

\section{Eye movements results}

The analyses conducted on test trials revealed that participants fixated inverted scenes $(\mathrm{M}=1849 \mathrm{~ms}, \mathrm{SE}=323 \mathrm{~ms})$ significantly longer than upright scenes $(\mathrm{M}=1693 \mathrm{~ms}, \mathrm{SE}=286$ $\mathrm{ms}), \mathrm{t}(6433.00)=5.32, \mathrm{p}<.001, \mathrm{dz}=1.024$, suggesting that more visual attention was allocated to inverted than upright scenes.

\section{Discussion of Experiment 3}

This third experiment aimed to confirm results of increased perceived sharpness of predictable relative to unpredictable stimuli implicitly assessed in Experiments 1 and 2 by using an explicit sharpness judgment task. To this end, participants had to decide which of two scenes (upright or inverted) was the blurriest, while both scenes were presented in most trials at an objectively equal level of blur. First, the analyses showed that most participants engaged in the task as they correctly perceived (more than $85 \%$ of the time) which scene was the blurriest when the two scenes had an objectively different blur level. Critically, when the two scenes had an objectively equal blur level, we observed that (1) participants did perceive a blur difference and that (2) they judged upright scenes as sharper than inverted scenes. These results further add support to the findings of Experiments 1 and 2 by showing that predictable scenes explicitly appear sharper than unpredictable ones. The analyses of eye movements additionally 
showed that more visual attention - as indexed by fixation time - was allocated to inverted than upright scenes. This result will be further discussed in the General Discussion.

Contrary to what was expected based on Experiments 1 and 2, participants did not necessarily judge the inverted scene as the blurriest one more often as the scene pairs became blurrier. We even observed the opposite trend, namely that participants judged the inverted scene as the blurriest one less frequently as the blur level of the scene pairs increased - and therefore as the precision decreased. One possible explanation for these results is that when the scenes are too blurry, the expectations would be too diverse and therefore too coarse to sharpen the representation of the stimulus. Alternatively, it is possible that when the blur level increased in the task, scenes were actually too blurred to be related to prior knowledge, even in the upright condition. Both the upright and inverted scenes therefore appeared unpredictable, thereby reducing the effect of predictability on perceived sharpness. These analyses according to the blur level of stimuli were conducted as a follow-up for the post-hoc analyses performed in Experiments 1 and 2 involving a perceptual matching task. However, in the context of the explicit judgement task used in Experiment 3, the proportion of upright/inverted scene selection as the blurriest may not be an adequate measure to assess how the effect of predictability on perceived sharpness of stimuli is influenced by their precision. Indeed, while this measure reflects the tendency to perceive one scene as sharper/blurrier than the other, it does not allow to quantify this effect (i.e., how big is the perceived difference in sharpness between the two scenes). For example, if the effect of predictability of stimuli on perceived sharpness is greater when stimuli have a medium/low than high precision, but that predictable stimuli are still perceived as sharper than unpredictable ones in all precision conditions, the proportion of selection of unpredictable stimuli as the blurriest may not necessarily differ across precision conditions.

\section{General Discussion}




\section{PRIOR SCENE KNOWLEDGE INCREASES ITS PERCEIVED SHARPNESS}

The present study investigated the influence of expectations based on prior experience and contextual information on scene and object perception. Based on prior studies (Alilović et al., 2021; Cheadle et al., 2015; de Lange et al., 2018; Han \& VanRullen, 2016; Kok et al., 2012; Lupyan, 2017; Perez et al., 2020; Stein \& Peelen, 2015; Teufel et al., 2018; Wyart et al., 2012) suggesting that expectations - or prediction signals - modulate the processing of information in visual areas by sharpening the representation of expected features of a stimulus, we hypothesized that predictable visual stimuli would be subjectively perceived as sharper than unpredictable ones. In Experiments 1 and 2, we used a perceptual blur matching task inspired by Lupyan (2017) to respectively address the effect of expectations on the perceived sharpness of objects and whole scenes. In both experiments, results suggested that at an objectively equal level of blur, predictable objects and scenes were subjectively perceived as sharper than unpredictable ones. Experiment 3 confirmed this conclusion using an explicit sharpness discrimination task. Post-hoc analyses of Experiment 1 and 2 additionally suggested that the effect of predictability on perceived sharpness of stimuli tended to increase as the precision of the visual signal decreased in line with the hypothesis of predictive coding theories (Friston, 2005; Rao \& Ballard, 1999).

Results of the present study therefore replicate previous findings by Lupyan (2017) that semantic knowledge affects the perceived sharpness of letter strings and allow to extend these findings to object and scene perception. Our results are also in agreement with recent findings of Perez et al. (2020) on object perception. In this study, the authors asked participants to perform comparative or equality judgments on the blurriness of familiar and novel objects. The novel objects were made from the same basic shapes composing familiar objects but assembled in a different configuration. Similarly to the present findings, they found that at an equal level of blur, participants judged familiar objects as sharper than novel ones. Overall, our and past studies support the hypothesis that expectations based on semantic knowledge, but also prior 


\section{PRIOR SCENE KNOWLEDGE INCREASES ITS PERCEIVED SHARPNESS}

experience and contextual information affect perception by making expected stimuli appear "sharper" and therefore influence "how we perceive".

Our sharpness adjustment measurements were used to address qualitative perceptual effects. How can we ascertain that they truly reflect the actual sharpness perception of participants instead of other non-perceptual effects? For example, Firestone \& Scholl (2016, see also Firestone \& Scholl, 2014) pointed out that the processing of any visual object not only involves the perception of its visual properties (e.g., its size, its color) but also making higherlevel judgments about it (e.g., its cost) which can bias perceptual reports. In the context of our study, it could therefore be argued that a predictable scene or object which can be more easily identified could be more readily judged as "sharp" while unpredictable stimuli would be judged as "blurry". In this case, the difference in reported perceived sharpness between predictable and unpredictable stimuli would reflect high-level judgments about stimuli rather than perceptual effects. If this was the case, such bias should also be observed in the identical pairtypes conditions. Predictable Samples should be more readily judged as sharp and they should be attributed a higher blur level than unpredictable Samples. Critically, this is not what we observed. The predictability effect was reduced in the identical pair-types trials compared to the different pair-type trials, arguing against an explanation of our results simply in terms of higher-level judgments. It has also been argued that expectations do not affect perception or early sensory processing but post-perceptual decisional processes involved in perceptual tasks (Bang \& Rahnev, 2017). In that sense, the tendency to perceive predictable stimuli as sharper than unpredictable ones would reflect a change in the decision criterion (i.e., response bias) rather than a perceptual bias. For example, when presented with blurred predictable Sample objects, participants could more easily "guess" the presence of sharp edges in the object based on contextual information and adapt their blur adjustment in consequence without it reflects an actual sharpened perception (e.g., they would add more blur than necessary because they 


\section{PRIOR SCENE KNOWLEDGE INCREASES ITS PERCEIVED SHARPNESS}

assume there are sharp edges without really perceiving these as sharp). However, such a bias should have also been present for predictable Samples in the identical pair-type trials but this is not what we observed. The results observed in these trials rule out an explanation by such a response bias. These considerations therefore make us feel confident that our findings are likely to reflect perceptual effects of expectations.

One could argue that this effect is due to attention rather than prior knowledge. Indeed, attention has been found to enhance the perception of attended stimuli for example resulting in increasing their perceived contrast (Carrasco et al., 2004) or spatial resolution (Yeshurun \& Carrasco, 1998). Therefore, increased perceived sharpness of predictable stimuli in our study could be explained by the fact that more attention was devoted to predictable than unpredictable stimuli. However, we believe this explanation is unlikely for several reasons. First, the tasks used in our experiments required participants to attend both the Target and Sample stimuli which were predictable or unpredictable in an equal number of trials. Therefore, if more attention was, for example, allocated to the Sample relative to the Target (or vice versa), the effects should be similar for predictable and unpredictable stimuli. It could also be argued that the effect is due to the difference in the task relevance between a predictable context and unpredictable context - as task relevance can be understood in terms of attention, it is possible that it influenced blur perception. However, participants' task was to focus on the blur adjustment (Experiments 1 and 2) or blur comparison (Experiment 3). The predictability was thus irrelevant to the task. Furthermore, several studies have shown that when it comes to object and scene perception, attention tends to be captured by novelty (Johnston et al., 1990; Park et al., 2010). In the context of our study, more attention should therefore have been allocated to unpredictable stimuli - which can be considered as novel since they were not related to a prior experience - than to predictable stimuli which were more familiar. This was supported by the analyses of fixation duration in Experiment 3, which showed that unpredictable stimuli elicited 


\section{PRIOR SCENE KNOWLEDGE INCREASES ITS PERCEIVED SHARPNESS}

longer fixation durations than predictable stimuli. Therefore, more attention to unpredictable stimuli can not explain the increased perceived sharpness of predictable stimuli observed in the present study. Finally, although Experiments 1 and 2 were run online on participants' personal computers and therefore in variable viewing conditions (e.g., in terms of viewing distance, screen size, luminance, contrast, etc.), we strongly believe that differences in terms of interindividual settings could not affect or explain our results. Indeed, the task required to compare and adjust the blur level of the Sample stimulus to match a Target stimulus simultaneously displayed on the same horizontal plane so that the two stimuli were always viewed and compared under the same conditions at each trial.

Increased perceived sharpness for expected stimuli observed in the present study supports the sharpening account of predictive coding theories (Alilović et al., 2021; Cheadle et al., 2015; de Lange et al., 2018; Han \& VanRullen, 2016; Kok et al., 2012; Lupyan, 2017; Perez et al., 2020; Stein \& Peelen, 2015; Teufel et al., 2018; Wyart et al., 2012). According to this account, expectations (or prediction signals) sent by high-level areas increase the sensitivity of neurons tuned to expected features in lower-level areas while suppressing the response of neurons tuned to unexpected ones. This results in a sharper representation of expected stimuli in these areas which would in turn lead to a sharper percept (de Lange et al., 2018; Han \& VanRullen, 2016; Kok et al., 2012; Teufel et al., 2018; Yon et al., 2018). Under this framework, results of Experiment 1 indicate that, although irrelevant to the task, contextual scene information was used to form prediction signals. These prediction signals could then have back-propagated from high- to low-order areas during object processing. In the case of predictable contextual information, this would, for example, allow to selectively enhance the response of neurons tuned to expected objects or shapes in areas of the ventral stream and of neurons tuned to the expected contours at the object location in early visual areas. This would in turn lead to a sharper percept of the object. In the case of unpredictable contextual 
information, prediction signals would not be precise enough leading to a noisier representation of the object in low-level cortical areas. Results of Experiments 2 and 3 on whole scene perception additionally suggest that sharpened representations based on contextual information not only apply to the processing of expected objects but more broadly to expected features in the entire scene.

This interpretation is also coherent with recent neuroimaging studies addressing the role of contextual information on visual processing. For example, Brandman and Peelen (2017) asked participants to categorize degraded objects presented alone or in a context (e.g., a bird flying over a lake, a tractor in a field) during an fMRI experiment. They examined the pattern of activity in object-selective areas of the ventral stream and found that the category of the object could be decoded with higher accuracy in these areas when they were presented in context than in isolation or when the context was presented without the object. This contextual effect in object-selective areas was associated with increased activity in scene-selective areas, suggesting that this area could be the origin of prediction signals about the object. More recently, Heilbron et al. (2020), presented participants with 5-letter words and pseudowords in an fMRI experiment. The middle letter of all letter strings was always an "N" or a "U". A decoding analysis of activity patterns in V1 and V2 revealed that the middle letter could be decoded with higher accuracy in these areas when it was embedded in a word than a pseudoword context. Overall, results from these studies are consistent with the view that expectations based on contextual information can enhance the representation of expected features in relatively high (i.e., object-selective areas) and low-level visual areas (i.e., V1 and V2).

While results of the present study support the sharpening account of predictive coding theories, they do not necessarily argue against the dampening account (de Lange et al., 2018; Kumar et al., 2017; Press et al., 2020; Richter et al., 2018). First, this account posits that prediction signals filter out predicted features of stimuli by silencing neurons tuned to these 


\section{PRIOR SCENE KNOWLEDGE INCREASES ITS PERCEIVED SHARPNESS}

features and increasing the sensitivity of neurons tuned to other features. Under this account, one would expect increased perceived sharpness of stimuli violating expectations. Critically, our experimental manipulations pertained to the presence or absence of informative content or prior knowledge allowing to form expectations via scrambling of contextual information or via scene inversion. These manipulations were chosen because they allowed us to control for lowlevel visual differences between the Target and Sample stimuli. However, they did not allow us to assess the effect of expectations validity as we did not include a condition in which stimuli could be unexpected/surprising and therefore violating expectations (e.g., an object in an intact but incongruent context). Such a condition would therefore be needed in further experiments in order to arbitrate between the sharpening and dampening accounts. Importantly, recent works suggest that these two accounts of predictive coding may not be mutually exclusive but could coexist (Press et al., 2020), so that their influence on perception would vary according to temporal constraints and signal precision. Under this framework, the sharpening mechanism would take place first, allowing to confirm expectations. The dampening mechanism would subsequently take over to boost the processing of the remaining unexpected features. This sequence would be further constrained by the signal characteristics. The sharpening mechanism would predominate when we have strong expectations but the visual signal is noisy. Predicted features would weigh more while unpredicted ones would be suppressed. The dampening mechanism would only take over when the signal is reliable but strongly diverges from expectations. Unpredicted features eliciting surprise would then weigh more while predicted features would be suppressed. In this context, increased perceived sharpness of predictable stimuli in our study could therefore reflect a predominance of the sharpening mechanism in order to process noisy (blurred) stimuli. This assumption is supported by post-hoc analyses of Experiments 1 and 2 suggesting that the effect of predictability on perceived sharpness increases with increasing blur level of the Target. Results of Experiment 3 however suggest the 


\section{PRIOR SCENE KNOWLEDGE INCREASES ITS PERCEIVED SHARPNESS}

processing of stimuli that are too noisy and for which expectations are too coarse may not benefit from this sharpening mechanism.

Another possibility which has been put forward in the literature is that expectations do not modulate early sensory response but later processes related to decision making and response selection (e.g., Bang \& Rahnev, 2017; Rungratsameetaweemana et al., 2018; Rungratsameetaweemana \& Serences, 2019; see however Aitken et al., 2020 for recent arguments against this view). Under this account, sharpened perception of predictable stimuli in the present study could result from a reweighting or selective attention to predicted features of stimuli occurring at later processing stages. Unfortunately, the present study cannot elucidate whether sharpened perception of predictable stimuli results from a modulation of early sensory or later cognitive processes. As previously discussed, our findings nevertheless provide evidence that expectations influence subjective perception, whether at early or late perceptual processing stages.

An important remaining question is whether sharper perception of expected visual features explains the effects of expectations in facilitating visual recognition, for example, in terms of recognition speed or accuracy as reported in many behavioural studies (Brandman \& Peelen, 2017; Davenport \& Potter, 2004; Greene et al., 2015; Joubert et al., 2007, 2008; Palmer, 1975; Richter et al., 2018; Roux-Sibilon et al., 2019). Could expected stimuli be recognized faster or more accurately because they are perceived better? Since the present study did not explicitly involve the recognition of scenes and objects, this assumption is only speculative and to our knowledge, no study has directly addressed this question so far. Further experiments using an explicit recognition task and response time recordings could enrich this point. In the context of our study, it could for example be hypothesized that a blurred predictable stimulus would be recognized faster than an unpredictable stimulus with a similar or even lower blur level. In any case, findings from fMRI studies support the assumption that better perception 


\section{PRIOR SCENE KNOWLEDGE INCREASES ITS PERCEIVED SHARPNESS}

and better recognition performances are correlated. For example, in Kok et al. (2012) sharpened representations of the stimuli in the visual cortex based on expectations were associated with higher behavioural performances (although no causal link could be established based on correlational analyses). Interestingly, a recent study by Gandolfo and Downing (2019) directly addressed the causal relationship between expectations and visual recognition performance, as well as the related cortical activity. In their study, the authors asked participants to categorize images of bodies (heavy vs. slim) and scenes (upright vs. inverted). These stimuli were preceded by a valid or an invalid cue which was orthogonal to the categorization task but could carry useful information to facilitate the categorization. For example, the cue for a body image could be either the "m" (i.e., for male) or the "f" letter (i.e., for female) implicitly associated with a heavy or a slim body, respectively. Results showed that a valid cue improved the categorization performances of both bodies and scenes. Then, during the cue presentation, the brain activity in body-selective or scene-selective cortical areas was disrupted via transcranial magnetic stimulation (TMS). Critically, the facilitation effect by a valid cue disappeared when the TMS was applied on body- or scene-selective areas during the cue presentation for the body or scene task, respectively. These results therefore indicated that expectation-based activity in these areas is directly related to categorization performance. Overall, these studies support the idea that the expression of expectations in visual areas facilitates visual recognition. Further studies would be needed to address whether higher recognition performances for expected visual stimuli are also associated with their increased perceived sharpness.

Our results also more broadly add support to the "cognitive penetrability of perception" theoretical framework (Lupyan, 2015; O'Callaghan et al., 2017; For arguments against this view, see Firestone \& Scholl, 2016; Pylyshyn, 1999) according to which perception not only affects one's cognitive state or knowledge but is also constrained by it. In this context, our findings are coherent with previous works suggesting that expectations or semantic knowledge 


\section{PRIOR SCENE KNOWLEDGE INCREASES ITS PERCEIVED SHARPNESS}

influence other aspects of perception such as contrast and color perception (Han \& VanRullen, 2016; Lupyan, 2015), perceived duration of stimuli (Pariyadath \& Eagleman, 2007; Schindel et al., 2011; Schweitzer et al., 2017), or perceived clarity of speech (Sohoglu et al., 2014). For example, Han and VanRullen (2016) found that line-drawings of objects were perceived as more contrasted with respect to the background than the same line-drawings with random locations (meaningless stimulus). In the context of color perception, Lupyan (2015) found that objects associated with a strong color-prior (e.g., yellow bananas) were perceived as more vivid than objects of the same color without such a strong prior (e.g., a yellow t-shirt). In the same way, Hansen et al. (2006; see also Olkkonen et al., 2008; Witzel et al., 2011) conducted a perceptual matching task in which participants were required to adjust the color of objects with strong color priors (e.g., yellow bananas) and colored discs without any color prior until they appeared gray. They observed that participants adjusted colored objects toward their opponent color to perceive them as gray (e.g. a yellow banana was adjusted to be slightly blue) suggesting that when they were objectively gray, they still appeared in their original color. However, colored discs unrelated to color-prior were accurately adjusted to an objective grey level. These results therefore indicated that prior knowledge can also influence color appearance. In the context of the sharpening account of predictive coding, these results could be explained by an enhanced perception of expected features (e.g., colors) of stimuli. Perceptual matching tasks such as the one used in the present study adapted to different contexts or modalities (e.g., speech processing, tactile or auditory perception) and using other matching criteria (e.g., color or sound intensity, noise level) may be a useful tool to more broadly assess how cognition can modulate perception.

Importantly, such perceptual effects may also constitute a useful measure to assess the use of prior knowledge and expectations in specific populations and disorders. For example, disorders such as autism spectrum disorders or schizophrenia have been linked to an 


\section{PRIOR SCENE KNOWLEDGE INCREASES ITS PERCEIVED SHARPNESS}

insufficient or excessive influence of expectations on perception, respectively (e.g., Pellicano \& Burr, 2012; Powers et al., 2017; Sinha et al., 2014; Teufel et al., 2015; Van de Cruys et al., 2014). In the context of our study, one could for example expect that participants with autism would not necessarily perceive predictable stimuli as sharper than unpredictable ones. On the contrary, schizophrenic patients should need to add even more blur than control participants to a predictable stimulus to perceive it as equally blurred as an unpredictable one. Predictive mechanisms have also been shown to evolve over the lifespan. For example, it has been proposed that the accumulation of sensory experience with aging may lead to rely more strongly on predictive processes, which would also help compensate for concomitant sensory deficits (Gilbert \& Moran, 2016; Lai et al., 2020; Moran et al., 2014). Under this assumption, it can be expected that the effect of predictability on the perceived sharpness of stimuli should be greater in aged than young individuals.

\section{Conclusion}

In conclusion, the results of the present study showed that expectations based on prior experience and contextual information increase the perceived sharpness of scenes and objects they contain, thus supporting the sharpening account of predictive coding theories (de Lange et al., 2018; Han \& VanRullen, 2016; Kok et al., 2012; Teufel et al., 2018; Yon et al., 2018). Our findings allow us to further extend the previous results of Lupyan (2017) on word reading to the perception of whole scenes and objects, defining the sharpening mechanism as a more general principle of visual perception. Expectations and knowledge about the visual environment not only help us to understand it more easily, but also makes us perceive it better.

\section{Acknowledgments}

The authors would like to thank all the participants who contributed to the study. We also warmly thank Gary Lupyan as well as two other anonymous Reviewers for their helpful and 
PRIOR SCENE KNOWLEDGE INCREASES ITS PERCEIVED SHARPNESS

constructive comments on earlier versions of the manuscript. We also thank Amaury Velasco for his help with data acquisition of Experiment 3. 
PRIOR SCENE KNOWLEDGE INCREASES ITS PERCEIVED SHARPNESS

\section{References}

Aitken, F., Turner, G., \& Kok, P. (2020). Prior Expectations of Motion Direction Modulate Early Sensory Processing. The Journal of Neuroscience, 40(33), 6389-6397. https://doi.org/10.1523/JNEUROSCI.0537-20.2020

Alilović, J., Slagter, H. A., \& van Gaal, S. (2021). Subjective visibility report is facilitated by conscious predictions only. Consciousness and Cognition, 87, 103048. https://doi.org/10.1016/j.concog.2020.103048

Bang, J. W., \& Rahnev, D. (2017). Stimulus expectation alters decision criterion but not sensory signal in perceptual decision making. Scientific Reports, 7(1), 17072. https://doi.org/10.1038/s41598-017-16885-2

Bar, M. (2004). Visual objects in context. Nature Reviews Neuroscience, 5(8), 617-629. https://doi.org/10.1038/nrn1476

Bar, M. (2007). The proactive brain: using analogies and associations to generate predictions. Trends in Cognitive Sciences, 11(7), 280-289. https://doi.org/10.1016/j.tics.2007.05.005

Bates, D., Kliegl, R., Vasishth, S., \& Baayen, R. H. (2015). Parsimonious Mixed Models.

Bates, D., Mächler, M., Bolker, B., \& Walker, S. (2015). Fitting linear mixed-effects models using lme4. Journal of Statistical Software, 67(1), 1-48. https://doi.org/10.18637/jss.v067.i01

Blakemore, S.-J., Wolpert, D. M., \& Frith, C. D. (1998). Central cancellation of selfproduced tickle sensation. Nature Neuroscience, 1(7), 635-640. https://doi.org/10.1038/2870

Brainard, D. H. (1997). The Psychophysics Toolbox. Spatial Vision, 10(4), 433-436. https://doi.org/10.1163/156856897X00357

Brandman, T., \& Peelen, M. V. (2017). Interaction between scene and object processing revealed by human fMRI and MEG decoding. The Journal of Neuroscience, 37(32), 


\section{PRIOR SCENE KNOWLEDGE INCREASES ITS PERCEIVED SHARPNESS}

7700-7710. https://doi.org/10.1523/JNEUROSCI.0582-17.2017

Carrasco, M., Ling, S., \& Read, S. (2004). Attention alters appearance. Nature Neuroscience, 7(3), 308-313. https://doi.org/10.1038/nn1194

Cheadle, S., Egner, T., Wyart, V., Wu, C., \& Summerfield, C. (2015). Feature expectation heightens visual sensitivity during fine orientation discrimination. Journal of Vision, 15(14), 14. https://doi.org/10.1167/15.14.14

Clark, A. (2013). Whatever next? Predictive brains, situated agents, and the future of cognitive science. Behavioral and Brain Sciences, 36(3), 181-204. https://doi.org/10.1017/S0140525X12000477

Davenport, J. L. (2007). Consistency effects between objects in scenes. Memory \& Cognition, 35(3), 393-401. https://doi.org/10.3758/BF03193280

Davenport, J. L., \& Potter, M. C. (2004). Scene Consistency in Object and Background Perception. Psychological Science, 15(8), 559-564. https://doi.org/10.1111/j.09567976.2004.00719.x

de Lange, F. P., Heilbron, M., \& Kok, P. (2018). How do expectations shape perception? Trends in Cognitive Sciences, 22(9), 764-779. https://doi.org/10.1016/j.tics.2018.06.002

Feldman, H., \& Friston, K. J. (2010). Attention, uncertainty, and free-energy. Frontiers in Human Neuroscience, 4, 1-23. https://doi.org/10.3389/fnhum.2010.00215

Firestone, C., \& Scholl, B. J. (2014). “Top-Down” Effects Where None Should Be Found. Psychological Science, 25(1), 38-46. https://doi.org/10.1177/0956797613485092

Firestone, C., \& Scholl, B. J. (2016). Cognition does not affect perception: Evaluating the evidence for “top-down” effects. Behavioral and Brain Sciences, 39, e229. https://doi.org/10.1017/S0140525X15000965

Friston, K. (2005). A theory of cortical responses. Philosophical Transactions of the Royal Society B: Biological Sciences, 360(1456), 815-836. 


\section{PRIOR SCENE KNOWLEDGE INCREASES ITS PERCEIVED SHARPNESS}

https://doi.org/10.1098/rstb.2005.1622

Gandolfo, M., \& Downing, P. E. (2019). Causal evidence for expression of perceptual expectations in category-selective extrastriate regions. Current Biology, 29(15), 24962500.e3. https://doi.org/10.1016/j.cub.2019.06.024

Gilbert, J. R., \& Moran, R. J. (2016). Inputs to prefrontal cortex support visual recognition in the aging brain. Scientific Reports, 6, 1-9. https://doi.org/10.1038/srep31943

Greene, M. R., Botros, A. P., Beck, D. M., \& Fei-Fei, L. (2015). What you see is what you expect: rapid scene understanding benefits from prior experience. Attention, Perception, \& Psychophysics, 77(4), 1239-1251. https://doi.org/10.3758/s13414-015-0859-8

Greene, M. R., \& Oliva, A. (2009). Recognition of natural scenes from global properties: Seeing the forest without representing the trees. Cognitive Psychology, 58(2), 137-176. https://doi.org/10.1016/j.cogpsych.2008.06.001

Han, B., \& VanRullen, R. (2016). Shape perception enhances perceived contrast: evidence for excitatory predictive feedback? Scientific Reports, 6(1), 22944. https://doi.org/10.1038/srep22944

Hansen, T., Olkkonen, M., Walter, S., \& Gegenfurtner, K. R. (2006). Memory modulates color appearance. Nature Neuroscience, 9, 1367-1368. https://doi.org/10.1038/nn1794

Heilbron, M., Richter, D., Ekman, M., Hagoort, P., \& de Lange, F. P. (2020). Word contexts enhance the neural representation of individual letters in early visual cortex. Nature Communications, 11(1), 321. https://doi.org/10.1038/s41467-019-13996-4

Johnston, W. A., Hawley, K. J., Plewe, S. H., Elliott, J. M. G., \& DeWitt, M. J. (1990). Attention capture by novel stimuli. Journal of Experimental Psychology: General, 119(4), 397-411. https://doi.org/10.1037/0096-3445.119.4.397

Joubert, O. R., Fize, D., Rousselet, G. A., \& Fabre-Thorpe, M. (2008). Early interference of context congruence on object processing in rapid visual categorization of natural scenes. 


\section{PRIOR SCENE KNOWLEDGE INCREASES ITS PERCEIVED SHARPNESS}

Journal of Vision, 8(13), 11-11. https://doi.org/10.1167/8.13.11

Joubert, O. R., Rousselet, G. a, Fize, D., \& Fabre-Thorpe, M. (2007). Processing scene context: Fast categorization and object interference. Vision Research, 47(26), 32863297. https://doi.org/10.1016/j.visres.2007.09.013

Judd, C. M., Westfall, J., \& Kenny, D. A. (2012). Treating stimuli as a random factor in social psychology: A new and comprehensive solution to a pervasive but largely ignored problem. Journal of Personality and Social Psychology, 103(1), 54-69. https://doi.org/10.1037/a0028347

Kaiser, D., Quek, G. L., Cichy, R. M., \& Peelen, M. V. (2019). Object Vision in a Structured World. Trends in Cognitive Sciences, 23(8), 672-685. https://doi.org/10.1016/j.tics.2019.04.013

Kauffmann, L., Ramanoël, S., Peyrin, C., \& Greene, M. R. (2014). The neural bases of spatial frequency processing during scene perception. Frontiers in Integrative Neuroscience, 8 , 37. https://doi.org/10.3389/fnint.2014.00037

Kirchner, H., \& Thorpe, S. J. (2006). Ultra-rapid object detection with saccadic eye movements: Visual processing speed revisited. Vision Research, 46(11), 1762-1776. https://doi.org/10.1016/j.visres.2005.10.002

Kok, P., \& de Lange, F. P. (2015). Predictive coding in sensory cortex. In B. U. Forstmann \& E. J. Wagenmakers (Eds.), An Introduction to Model-Based Cognitive Neuroscience (pp. 221-244). Springer New York. https://doi.org/10.1007/978-1-4939-2236-9_11

Kok, P., Jehee, J. F. M., \& de Lange, F. P. (2012). Less is more: Expectation sharpens representations in the primary visual cortex. Neuron, 75(2), 265-270. https://doi.org/10.1016/j.neuron.2012.04.034

Kumar, S., Kaposvari, P., \& Vogels, R. (2017). Encoding of predictable and unpredictable stimuli by inferior temporal cortical neurons. Journal of Cognitive Neuroscience, 29(8), 


\section{PRIOR SCENE KNOWLEDGE INCREASES ITS PERCEIVED SHARPNESS}

1445-1454. https://doi.org/10.1162/jocn_a_01135

Kuznetsova, A., Brockhoff, P. B., \& Christensen, R. H. B. (2017). lmerTest package: Tests in linear mixed effects models. Journal of Statistical Software, 82(13), 1-26. https://doi.org/10.18637/jss.v082.i13

Lai, L. Y., Frömer, R., Festa, E. K., \& Heindel, W. C. (2020). Age-related changes in the neural dynamics of bottom-up and top-down processing during visual object recognition: an electrophysiological investigation. Neurobiology of Aging, 94, 38-49. https://doi.org/10.1016/j.neurobiolaging.2020.05.010

Lakens, D. (2013). Calculating and reporting effect sizes to facilitate cumulative science: A practical primer for t-tests and ANOVAs. Frontiers in Psychology, 4, 1-12. https://doi.org/10.3389/fpsyg.2013.00863

Lee, T. S., \& Mumford, D. (2003). Hierarchical Bayesian inference in the visual cortex. Journal of the Optical Society of America A, 20(7), 1434. https://doi.org/10.1364/JOSAA.20.001434

Lupyan, G. (2015). Object knowledge changes visual appearance: Semantic effects on color afterimages. Acta Psychologica, 161, 117-130. https://doi.org/10.1016/j.actpsy.2015.08.006

Lupyan, G. (2017). Objective effects of knowledge on visual perception. Journal of Experimental Psychology: Human Perception and Performance, 43(4), 794-806. https://doi.org/10.1037/xhp0000343

Moran, R. J., Symmonds, M., Dolan, R. J., \& Friston, K. J. (2014). The brain ages optimally to model its environment: Evidence from sensory learning over the adult lifespan. PLoS Computational Biology, 10(1), e1003422. https://doi.org/10.1371/journal.pcbi.1003422

Murray, S. O., Schrater, P., \& Kersten, D. (2004). Perceptual grouping and the interactions between visual cortical areas. Neural Networks, 17(5-6), 695-705. 


\section{PRIOR SCENE KNOWLEDGE INCREASES ITS PERCEIVED SHARPNESS}

https://doi.org/10.1016/j.neunet.2004.03.010

O’Callaghan, C., Kveraga, K., Shine, J. M., Adams, R. B., \& Bar, M. (2017). Predictions penetrate perception: Converging insights from brain, behaviour and disorder. Consciousness and Cognition, 47(5), 63-74. https://doi.org/10.1016/j.concog.2016.05.003

Oliva, A., \& Torralba, A. (2007). The role of context in object recognition. Trends in Cognitive Sciences, 11(12), 520-527. https://doi.org/10.1016/j.tics.2007.09.009

Olkkonen, M., Hansen, T., \& Gegenfurtner, K. R. (2008). Color appearance of familiar objects: Effects of object shape, texture, and illumination changes. Journal of Vision, 8(5), 13. https://doi.org/10.1167/8.5.13

Palmer, T. E. (1975). The effects of contextual scenes on the identification of objects. Memory \& Cognition, 3(5), 519-526. https://doi.org/10.3758/BF03197524

Pariyadath, V., \& Eagleman, D. (2007). The effect of predictability on subjective duration. PLoS ONE, 2(11), e1264. https://doi.org/10.1371/journal.pone.0001264

Park, J., Shimojo, E., \& Shimojo, S. (2010). Roles of familiarity and novelty in visual preference judgments are segregated across object categories. Proceedings of the National Academy of Sciences of the United States of America, 107(33), 14552-14555. https://doi.org/10.1073/pnas.1004374107/-

/DCSupplemental.www.pnas.org/cgi/doi/10.1073/pnas.1004374107

Pelli, D. G. (1997). The VideoToolbox software for visual psychophysics: transforming numbers into movies. Spatial Vision, 10(4), 437-442. https://doi.org/10.1163/156856897X00366

Pellicano, E., \& Burr, D. (2012). When the world becomes too real : a Bayesian explanation of autistic perception. Trends in Cognitive Sciences, 16(10), 504-510. https://doi.org/10.1016/j.tics.2012.08.009 


\section{PRIOR SCENE KNOWLEDGE INCREASES ITS PERCEIVED SHARPNESS}

Perez, D. C., Cook, S. M., \& Peterson, M. A. (2020). Prior experience alters the appearance of blurry object borders. Scientific Reports, 10(1), 1-13. https://doi.org/10.1038/s41598$020-62728-y$

Powers, A. R., Mathys, C., \& Corlett, P. R. (2017). Pavlovian conditioning - induced hallucinations result from overweighting of perceptual priors. Science, 357(6351), 596600. https://doi.org/10.1126/science.aan3458

Press, C., Kok, P., \& Yon, D. (2020). The perceptual prediction paradox. Trends in Cognitive Sciences, 24(1), 13-24. https://doi.org/10.1016/j.tics.2019.11.003

Pylyshyn, Z. (1999). Is vision continuous with cognition? The case for cognitive impenetrability of visual perception. Behavioral and Brain Sciences, 22, 341-423. https://doi.org/10.1017/s0140525x99002022

R Core Team. (2019). R: A language and environment for statistical computing (3.6.1). $\mathrm{R}$ Foundation for Statistical Computing.

Rao, R. P. N., \& Ballard, D. H. (1999). Predictive coding in the visual cortex: A functional interpretation of some extra-classical receptive-field effects. Nature Neuroscience, 2, 79-87. https://doi.org/10.1038/4580

Richter, D., Ekman, M., \& de Lange, F. P. (2018). Suppressed sensory response to predictable object stimuli throughout the ventral visual stream. Journal of Neuroscience, 38(34), 7452-7461. https://doi.org/10.1523/JNEUROSCI.3421-17.2018

Rousselet, G. A., Joubert, O. R., \& Fabre-Thorpe, M. (2005). How long to get to the "gist" of real-world natural scenes? Visual Cognition, 12(6), 852-877. https://doi.org/10.1080/13506280444000553

Roux-Sibilon, A., Trouilloud, A., Kauffmann, L., Guyader, N., Mermillod, M., \& Peyrin, C. (2019). Influence of peripheral vision on object categorization in central vision. Journal of Vision, 19(14), 1-16. https://doi.org/10.1167/19.14.7 


\section{PRIOR SCENE KNOWLEDGE INCREASES ITS PERCEIVED SHARPNESS}

Rungratsameetaweemana, N., Itthipuripat, S., Salazar, A., \& Serences, J. T. (2018).

Expectations Do Not Alter Early Sensory Processing during Perceptual Decision-

Making. The Journal of Neuroscience, 38(24), 5632-5648.

https://doi.org/10.1523/JNEUROSCI.3638-17.2018

Rungratsameetaweemana, N., \& Serences, J. T. (2019). Dissociating the impact of attention and expectation on early sensory processing. Current Opinion in Psychology, 29, 181186. https://doi.org/10.1016/j.copsyc.2019.03.014

Schindel, R., Rowlands, J., \& Arnold, D. H. (2011). The oddball effect: Perceived duration and predictive coding. Journal of Vision, 11(2), 17. https://doi.org/10.1167/11.2.1

Schweitzer, R., Trapp, S., \& Bar, M. (2017). Associated information increases subjective perception of duration. Perception, 46(8), 1000-1007. https://doi.org/10.1177/0301006616689579

Schyns, P. G., \& Oliva, A. (1994). From blobs to boundary edges: Evidence for time- and spatial-scale-dependent scene recognition. Psychological Science, 5(4), 195-200. https://doi.org/10.1111/j.1467-9280.1994.tb00500.x

Sinha, P., Kjelgaard, M. M., Gandhi, T. K., Tsourides, K., \& Cardinaux, A. L. (2014). Autism as a disorder of prediction. Proceedings of the National Academy of Sciences of the United States of America, 111(42), 15220-15225. https://doi.org/10.1073/pnas.1416797111

Sohoglu, E., Peelle, J. E., Carlyon, R. P., \& Davis, M. H. (2014). Top-down influences of written text on perceived clarity of degraded speech. Journal of Experimental Psychology: Human Perception and Performance, 40(1), 186-199. https://doi.org/10.1037/a0033206

Spratling, M. W. (2017). A hierarchical predictive coding model of object recognition in natural images. Cognitive Computation, 9(2), 151-167. https://doi.org/10.1007/s12559- 


\section{PRIOR SCENE KNOWLEDGE INCREASES ITS PERCEIVED SHARPNESS}

016-9445-1

Stein, T., \& Peelen, M. V. (2015). Content-specific expectations enhance stimulus detectability by increasing perceptual sensitivity. Journal of Experimental Psychology: General, 144(6), 1089-1104. https://doi.org/10.1037/xge0000109

Summerfield, C., \& de Lange, F. P. (2014). Expectation in perceptual decision making: neural and computational mechanisms. Nature Reviews Neuroscience, 15(11), 745-756. https://doi.org/10.1038/nrn3838

Summerfield, C., \& Egner, T. (2009). Expectation (and attention) in visual cognition. Trends in Cognitive Sciences, 13(9), 403-409. https://doi.org/10.1016/j.tics.2009.06.003

Teufel, C., Dakin, S. C., \& Fletcher, P. C. (2018). Prior object-knowledge sharpens properties of early visual feature-detectors. Scientific Reports, 8, 1-12. https://doi.org/10.1038/s41598-018-28845-5

Teufel, C., \& Fletcher, P. C. (2020). Forms of prediction in the nervous system. Nature Reviews Neuroscience, 21(4), 231-242. https://doi.org/10.1038/s41583-020-0275-5

Teufel, C., Subramaniam, N., Dobler, V., Perez, J., Finnemann, J., Mehta, P. R., Goodyer, I. M., \& Fletcher, P. C. (2015). Shift toward prior knowledge confers a perceptual advantage in early psychosis and psychosis-prone healthy individuals. Proceedings of the National Academy of Sciences of the United States of America, 112(43), 1340113406. https://doi.org/10.1073/pnas.1503916112

Thorpe, S. J., Fize, D., \& Marlot, C. (1995). Categorisation times for natural images. Perception, 24, 8-8.

Torralba, A. (2003). Contextual priming for object detection. International Journal of Computer Vision, 53(2), 169-191. https://doi.org/10.1023/A:1023052124951

Van de Cruys, S., Evers, K., Van der Hallen, R., Van Eylen, L., Boets, B., De-Wit, L., \& Wagemans, J. (2014). Precise minds in uncertain worlds : Predictive coding in autism. 


\section{PRIOR SCENE KNOWLEDGE INCREASES ITS PERCEIVED SHARPNESS}

Psychological Review, 121(4), 649-675. https://doi.org/10.1037/a0037665

Witzel, C., Valkova, H., Hansen, T., \& Gegenfurtner, K. R. (2011). Object Knowledge

Modulates Colour Appearance. I-Perception, 2(1), 13-49. https://doi.org/10.1068/i0396

Wyart, V., Nobre, A. C., \& Summerfield, C. (2012). Dissociable prior influences of signal probability and relevance on visual contrast sensitivity. Proceedings of the National Academy of Sciences of the United States of America, 109(9), 3593-3598. https://doi.org/10.1073/pnas.1204601109

Yeshurun, Y., \& Carrasco, M. (1998). Attention improves or impairs visual performance by enhancing spatial resolution. Nature, 396(6706), 72-75. https://doi.org/10.1038/23936

Yon, D., Gilbert, S. J., de Lange, F. P., \& Press, C. (2018). Action sharpens sensory representations of expected outcomes. Nature Communications, 9(1), 1-8. https://doi.org/10.1038/s41467-018-06752-7 